\title{
A transcendental view on the continuum: Woodin's conditional platonism
}

\author{
Jean PETITOT*
}

December 2007

To appear in Intellectica.

\begin{abstract}
One of the main difficulty concerning the nature of the continuum is to do justice, inside the set theoretical Cantorian framework, to the classical conception (from Aristotle to Thom, via Kant, Peirce, Brentano, Husserl and Weyl) according to which the continuum is a non-compositional, cohesive, primitive, and intuitive datum. This paper investigates such possibilities, from Gödel to Woodin, of modelling inside a $Z F C$-universe the transcendence of the intuitive continuum w.r.t. its symbolic determination.

Keywords: constructive universe, continuum, Gödel, forcing, Kant, large cardinals, $\Omega$-logic, projective hierarchy, $V=L$, Woodin, $0^{\#}$.

\section{Introduction}

One of the main general philosophical problem raised by the nature of the continuum is the conflict between two traditions: the older one, which can be called "neo-Aristotelian", even if this sounds rather vague, and the now the classical one, namely the Cantorian tradition. According to the neo-Aristotelian tradition, the continuum is experienced and thought of as a non-compositional, cohesive, primitive, and
\end{abstract}

*EHESS \& CREA (Ecole Polytechnique), France. petitot@ehess.fr. 
intuitive datum. It can be segmented into parts but these parts are themselves continua and points are only their boundaries.

This point of view was very well defended by Kant. As soon as in his 1770 Dissertatio, he emphasized the fact that

"a magnitude is continuous when it is not composed out of simple elements" (AK, II, p. 399 1),

and explained that for the continuous "pure intuitions" of space and time

"any part of time is still a time, and the simple elements which are in time, namely the moments, are not parts but limits between which a time takes place" (AK, II, p. 399). "space must necessarily be conceived of as a continuous magnitude, (...) and therefore simple elements in space are not parts but limits" (AK, II, p. 404).

He wrote also in the "Anticipations of perception" of the Critic of Pure Reason:

"Space and time are quanta continua because no part of them can be given without being enclosed into limits (points or moments) (...). Space is made up only of spaces and time of times. Points and moments are only limits, that is to say, simple places bounding space and time (...), and neither space nor time can be made up of simple places, that is of integral parts which would be given before space and time themselves" (AK, III, p. 154).

We see that this conception of the continuum is based on mereological properties, and especially on the concept of boundary (Grenze): points are boundaries and boundaries are dependent entities which cannot exist independently of the entities they bound.When there are no explicit boundaries, the continuum is characterized by the "fusion" of its parts 2 Moreover, Leibniz's principle of continuity holds (every function is continuous).

\footnotetext{
${ }^{1}$ AK refers to the collected works Kants gesammelte Schriften, Preussische Akademie der Wissenschaften.

${ }^{2}$ It is very easy to construct a model of such a mereology. Let us take $\mathbb{R}$ with its standard topology and posit that the only admissible parts $U$ of $\mathbb{R}$ are its open subsets $U \in \mathcal{P}_{\text {ad }}(\mathbb{R})$. Let $U, V \in \mathcal{P}_{\text {ad }}(\mathbb{R})$. The complement $\neg U$ of $U$ is the interior $\operatorname{Int}(\bar{U})$ of its classical complement $\bar{U}$ (which is a closed subset). Therefore $U \cap \neg U=\emptyset$ but $U \cup \neg U \neq \mathbb{R}$. Conversely; if $U \cup V=\mathbb{R}$ then $U \cap V \neq \emptyset$. This means that, for the open mereology, $\mathbb{R}$ is
} 
Even after the Cantor-Dedekind arithmetization, the "neo-Aristotelian" non-compositionality of the continuum kept on raising fondamental problems for some of the greatest philosophers, mathematicians, and psychologists, such as Peirce, Brentano, Stumpf (who elaborated the key concept of "fusion": Verschmelzung), Husserl 3, or Thom (for whom the continuum possessed an ontological primacy as a qualitative homogeneous Aristotelian "homeomer"). Gödel himself considered the real intuition of the continuum in this way and opposed it to its set theoretical idealisation.

Peirce developed a "synechology", "syneche" being the greek term for "continuum". Mathematically, he was also the first, as far as I know, to reject the continuum hypothesis $\mathrm{CH}\left(2^{\aleph_{0}}=\aleph_{1}\right)$ and to define the power $\mathfrak{c}$ of the continuum as a large cardinal, namely an inaccessible cardinal (if $\kappa<\mathfrak{c}$ then $2^{\kappa}<\mathfrak{c}$ ). In some texts, Peirce even explained that the continuum could be so huge that it would fail to be a cardinal.

Husserl was the first, in the third Logical Investigation, to formalize the idea of a mereology, and, after him, Stanisław Lesniewski developped the theory between 1916 and 1921. But the definition of boundaries in a mereo(topo)logy remained up to now highly problematic as it is argued, e.g., in Breysse-De Glas (2007).

All these conceptions develop the same criticism against the idea of arithmetizing the continuum. According to them, the continuum can be measured using systems of numbers, but no system can exhaust the substratum it measures. They consider:

1. that a point in the continuum is a discontinuity (a mark, a local heterogeneity, a boundary) which is like a singular individuated "atom" which can be refered to by a symbol;

2. that quantified sentences of an appropriate predicate calculus can be therefore interpreted in the continuum;

3. that systems of numbers can of course measure such systems of marks and enable their axiomatic control;

4. but that the arithmetization of the continuum postulates, what's more, that the intuitive phenomenological continuum is reducible to such a set-theoretic system (Cantor-Dedekind);

undecomposable. Topological boundaries $\partial U$ are not admissible parts but only limits, and bound both $U$ and $\neg U$. Heyting used this mereotopology for defining truth in intuitionist logic.

${ }^{3}$ Concerning the concept of Verschmelzung in Stumpf and Husserl, see. Petitot [1994]. 
5. and therefore that such a reductive arithmetization is unacceptable for it violates the original intuitive mode of givenness of the continuum.

Let us leave phenomenology and psychology for mathematics. Even if we adopt a set-theoretic perspective making the continuum a set, non-compositionality and cohesivity remain meaningful. They now mean in particular that the continuum cannot be identified with a set of well individuated points. It is the case in intuitionistic logic where the law of the excluded middle, which implies that two elements $a$ and $b$ of $\mathbb{R}$ are different or equal, and the law of comparability, which implies $a=b$, or $a<b$, or $a>b$, are no longer valid. For Hermann Weyl (1918), this intrinsic lack of individuation and localization of points in $\mathbb{R}$ characterizes the continuum as an intuitive datum.

Given the close link discovered by Bill Lawvere between intuitionistic logic and topos theory, it is not surprising that in many topoï the object $\mathbb{R}$ is undecomposable and all the morphisms $f: \mathbb{R} \rightarrow \mathbb{R}$ are continuous (Leibniz's principle, see e.g. John Bell's works 4 ).

But, even in the realm of classical logic and classical set theory (that is $Z F C$ : Zermelo-Fraenkel + axiom of choice) there exist many evidences of the transcendence of the continuum relatively to its symbolic logical control. Of course, they occur in non-standard models of $\mathbb{R}$, but these non-Archimedian models are not well-founded and will not be analyzed here 5 I will rather focus on their status in wellfounded models $V$ of $Z F C$.

So, the context of this paper will be the theory of the continuum in universe of sets satisfying $Z F C$. We will see that, even in this purely formal framework, the Kantian opposition between "intuitive" and "conceptual" remains operating, where "conceptual" now refers to the logical control and symbolic determination of the continuum (as it was already the case for Kant himself who considered arithmetic, contrary to geometry, as "conceptual" and "intellectual", and algebra as a calculus on "symbolic constructions").6 The key question remains the same: is it possible to determine completely the intuitive continuum using logical symbolic constructions?

We will meet in the sequel an alternative opposing two different

\footnotetext{
${ }^{4}$ See in particular Bell's contribution to this volume.

${ }^{5}$ For an introduction to non-standard analysis, see Petitot [1979], [1989] and their bibliographies.

${ }^{6}$ Critic of Pure Reason, "Transcendental Methodology", AK, III, p. 471.
} 
types of philosophies.

1. Philosophies of the first type are "minimizing", "ontologically" deflationist (in the sense of restricting what axioms of existence are admissible), nominalist, and constructive. They consider that the only meaningful content of the continuum is the part which can be "conceptually" (that is symbolically) well determined and that the rest is "inherently vague". A celebrated representative of such a perspective is Solomon Feferman who considers that the continuum cannot be a definite mathematical object since some of its properties, such as Cantor's continuum hypothesis, are not expressible by definite propositions. But, as explained by John Steel (2004) in a criticism of Feferman:

"Taken seriously, this analysis leads us into a retreat to some much weaker constructivist language, a retreat which would toss out good mathematics in order to save inherently vague philosophy."

2. It is why, philosophies of the second type are, on the contrary, "maximizing", "ontologically" inflationist, platonist in a sophisticated sense, and highly non constructive. They aim at modelling inside a $Z F C$-universe the transcendence of the intuitive continuum w.r.t. its logical symbolic mastery. Owing to this, they must introduce non constructive axioms for higher infinite.

\section{Preliminaries 7}

\subsection{Axioms}

We work in $Z F C$ with the classical axioms:

Extensionality: sets are determined by their elements,

Pairing: the pair $\{a, b\}$ exists for every sets $a$ and $b$,

Union: the union $\bigcup X=\{u \in x \in X\}$ of every set $X$ exists,

Power set: the set $\mathcal{P}(X)=\{u \subseteq X\})$ of subsets of every set $X$ exists,

Comprehension or Separation (axiom schema): if $\varphi(x)$ is a formula, the subset $\{x \in X: \varphi(x)\}$ exists for every set $X$,

\footnotetext{
${ }^{7}$ See Jech [1978].
} 
Replacement (axiom schema): if $y=f(x)$ is function (i.e. a relation $\varphi(x, y)$ s.t. $\varphi(x, y)$ and $\varphi(x, z)$ imply $y=z)$, then the image $\{f(x): x \in X\}$ exists for every set $X$,

Infinity: there exists an inductive set, that is a set $I$ s.t. $\emptyset \in I$ and if $x \in I$ then $x \cup\{x\} \in I$,

Regularity: all sets have minimal $\in$-elements,

Choice: every family of sets $X_{s}, s \in S$, has a choice function $f: S \rightarrow \mathcal{P}(X)$ s.t. $f(s) \in X_{s}$ for every $s \in S$ (this axiom of existence doesn't define any specific $f$ and is highly non constructive).

\subsection{Ordinals and cardinals}

Ordinals are the sets that are $\in$-transitive $(y \in X$ implies $y \subset X$, that is $\bigcup X \subseteq X$ or $X \subset \mathcal{P}(X))$ and well-ordered by $\in$. All well-ordered sets are order-isomorphic to an ordinal. Every ordinal is a successor: $\alpha=\beta+1$ or a limit ordinal $\alpha=\operatorname{Sup}(\beta: \beta<\alpha)$ (and then $\forall \beta<\alpha$, $\beta+1<\alpha$ ). A limit ordinal is like an "horizon" for enumeration: it is impossible to reach its limit in a finite number of steps. The smallest limit ordinal is $\omega=\mathbb{N}$. The sum of two ordinals is their concatenation (non commutativity: $1+\omega=\omega \neq \omega+1$ ). The product of two ordinals $\alpha . \beta$ is $\beta$-times the concatenation of $\alpha$ (lexicographic order) (non commutativity: $2 . \omega=\omega \neq \omega \cdot 2=\omega+\omega$ ). An ordinal $\alpha$ is a limit ordinal iff there exists $\beta$ s.t. $\alpha=\omega . \beta$.

Cardinals $|X|$ are equivalence classes of the equivalence relation of equipotence: $X$ eq $Y$ if there exists a bijective (i.e. one-to-one onto) map $f: X \rightarrow Y$. They highly depend upon the functions existing in the $Z F C$-universe under consideration.

Cantor theorem. $|X|<|\mathcal{P}(X)|$.

Indeed, let $f: X \rightarrow \mathcal{P}(X)$. Then $Y=\{x \in X: x \notin f(x)\}$ exists (Comprehension axiom). But $Y \notin f(X)$ for if it would exist $z \in X$ with $f(z)=Y$, then $z \in Y \Leftrightarrow z \notin Y$. Contradiction.

If $|A|=\kappa$, then $|\mathcal{P}(A)|=2^{\kappa}$ since $X \subseteq A$ is equivalent to its characteristic function $\chi_{X}: A \rightarrow\{0,1\}$ and $\chi_{X} \in 2^{\kappa}$. Cantor theorem implies therefore $\kappa<2^{\kappa}$.

For ordinals, cardinals numbers are the $\alpha$ s.t. $|\beta|<|\alpha|$ for every $\beta<\alpha$. They are the minimal elements in the equivalence classes of equipotent ordinals. So every infinite cardinal is a limit ordinal. Every well-ordered set has such a cardinal number for cardinal. These cardinal numbers are the alephs $\aleph_{\alpha}$. Each $\aleph_{\alpha}$ has a successor, namely $\aleph_{\alpha}^{+}=\aleph_{\alpha+1}$. If $\alpha$ is a limit ordinal, $\aleph_{\alpha}=\omega_{\alpha}=\operatorname{Sup}_{\beta<\alpha}\left(\omega_{\beta}\right)$. 
Theorem. For alephs, the sum and product operations are trivial: $\aleph_{\alpha}+\aleph_{\beta}=\aleph_{\alpha} \aleph_{\beta}=\max \left(\aleph_{\alpha}, \aleph_{\beta}\right)$ (the bigger takes all).

A consequence is that if $\alpha \leq \beta$ then $\aleph_{\alpha}^{\aleph_{\beta}}=2^{\aleph_{\beta}}$. Indeed, $2^{\aleph_{\beta}} \leq$ $\aleph_{\alpha}^{\aleph_{\beta}} \leq\left(2^{\aleph_{\alpha}}\right)^{\aleph_{\beta}}=2^{\aleph_{\alpha} \cdot \aleph_{\beta}}=2^{\aleph_{\beta}}$. But other exponentiations raise fundamental problems.

Under the axiom of choice $A C$, every set can be well-ordered and therefore all cardinals are alephs. In particular $2^{\aleph_{0}}$ is an aleph $\aleph_{\alpha}$.

\section{The underdetermination of cardinal arithmetic in $Z F C$}

Let $V$ be a universe of set theory (i.e. a model of $Z F C$ ). We work in $\mathbb{R}$ or in the isomorphic Baire space $\mathcal{N}=\omega^{\omega}$. The first limit we meet is that the axioms of $Z F C$ are radically insufficient for determining the cardinal arithmetic of $V$ as it is clearly shown by the following celebrated result 8

\subsection{Easton theorem}

For every ordinal $\alpha$ let $F(\alpha)$ be the power function defined by $2^{\aleph_{\alpha}}=$ $\aleph_{F(\alpha)} 9$ One can show that:

1. $F$ is a monotone increasing function: if $\alpha \leq \beta$ then $F(\alpha) \leq F(\beta)$;

2. König's law: $\operatorname{cf}\left(\aleph_{F(\alpha)}\right)>\aleph_{\alpha}$, where the cofinality $\operatorname{cf}(\alpha)$ of an ordinal $\alpha$ is defined as the smallest cardinality $\chi$ of a cofinal (i.e. unbounded) subset $X$ of $\alpha$ (i.e. $\operatorname{Sup} X=\alpha$ ). For instance, $\operatorname{cf}(\omega+\omega)=\operatorname{cf}\left(\aleph_{\alpha+\omega}\right)=\omega$. Of course, $\operatorname{cf}(\alpha)$ is a limit ordinal and $\operatorname{cf}(\alpha) \leq \alpha$. The cardinal $\kappa$ is called regular if $\operatorname{cf}(\kappa)=\kappa$ i.e. if, as far as we start with $\alpha<\kappa$, it is impossible to reach the horizon of $\kappa$ in less than $\kappa$ steps. In some sense the length of $\kappa$ is equal to its "asymptotic" length and cannot be exhausted before reaching the horizon. $\operatorname{As} \operatorname{cf}(\operatorname{cf}(\kappa))=\operatorname{cf}(\kappa), \operatorname{cf}(\kappa)$ is always regular. As cf $\left(\aleph_{\alpha+\omega}\right)=\omega, \aleph_{\alpha+\omega}$ is always singular 10

\footnotetext{
${ }^{8}$ See Jacques Stern [1976] for a presentation.

${ }^{9}$ More generally one can consider the power function $(\lambda, \kappa) \mapsto \lambda^{\kappa}$ for each pair $(\lambda, \kappa)$ of cardinals.

${ }^{10}$ So a cardinal $\kappa$ is singular iff $\kappa=\bigcup_{i \in I} \alpha_{i}$ with $|I|<\kappa$ and $\left|\alpha_{i}\right|<\kappa \forall i \in I$.
} 
König's law is a consequence of a generalization of Cantor theorem which says that $1+1+\ldots(\kappa$ times $)<2.2 \ldots(\kappa$ times $)$ : if $\kappa_{i}<\lambda_{i}$ $\forall i \in I$, then $\sum_{i \in I} \kappa_{i}<\prod_{i \in I} \lambda_{i}$. Let $\kappa_{i}<2^{\aleph_{\alpha}}$ and $\lambda_{i}=2^{\aleph_{\alpha}}$ for $i<\omega_{\alpha}$. Then $\sum_{i<\omega_{\alpha}} \kappa_{i}<\prod_{i<\omega_{\alpha}} \lambda_{i}=\left(2^{\aleph_{\alpha}}\right)^{\aleph_{\alpha}}=2^{\aleph_{\alpha}}$. Therefore with an $\omega_{\alpha}$ sequence of $\kappa_{i}<2^{\aleph_{\alpha}}$ it is impossible to get $\operatorname{Sup}\left(\kappa_{i}\right)=2^{\aleph_{\alpha}}$ since for infinite cardinals $\operatorname{Sup}=\sum$.

An immediate corollary of König's law is

Theorem. If $\kappa$ is an infinite cardinal, $\kappa<\kappa^{\mathrm{cf}(\kappa)}$ (compare with Cantor: $\left.\kappa<2^{\kappa}\right)$.

Indeed, if $\kappa_{i}<\kappa$ for $i<\operatorname{cf}(\kappa)$ and $\kappa=\operatorname{Sup}\left(\kappa_{i}\right)=\sum_{i<\operatorname{cf}(\kappa)} \kappa_{i}$ then $\kappa=\sum_{i<\operatorname{cf}(\kappa)} \kappa_{i}<\prod_{i<\operatorname{cf}(\kappa)} \kappa=\kappa^{\operatorname{cf}(\kappa)}$.

In fact one can prove that the essential cardinals for cardinal arithmetic are the $2^{\kappa}$ and the $\kappa^{\mathrm{cf}(\kappa)}$ (Gimel function). They enable to compute all the $\aleph_{\alpha}^{\aleph_{\beta}}$ :

\section{Theorem.11}

1. If $\alpha \leq \beta$, then $\aleph_{\alpha}^{\aleph_{\beta}}=2^{\aleph_{\beta}}$.

2. If $\alpha>\beta$ and $\exists \gamma<\alpha$ s.t. $\aleph_{\gamma}^{\aleph_{\beta}} \geq \aleph_{\alpha}$, then $\aleph_{\alpha}^{\aleph_{\beta}}=\aleph_{\gamma}^{\aleph_{\beta}}$.

3. If $\alpha>\beta$ and $\forall \gamma<\alpha$ we have $\aleph_{\gamma}^{\aleph_{\beta}}<\aleph_{\alpha}$ then

(a) if $\aleph_{\alpha}$ is regular or $\operatorname{cf}\left(\aleph_{\alpha}\right)>\aleph_{\beta}$ then $\aleph_{\alpha}^{\aleph_{\beta}}=\aleph_{\alpha}$;

(b) if $\operatorname{cf}\left(\aleph_{\alpha}\right) \leq \aleph_{\beta}<\aleph_{\alpha}$ then $\aleph_{\alpha}^{\aleph_{\beta}}=\aleph_{\alpha}^{\mathrm{cf}\left(\aleph_{\alpha}\right)}$.

If the generalized continuum hypothesis $(G C H)$ holds, König's law is trivial because $F(\alpha)=\alpha+1$, every cardinal $\aleph_{\alpha+1}$ is regular and therefore $\operatorname{cf}\left(\aleph_{\alpha+1}\right)=\aleph_{\alpha+1}>\aleph_{\alpha}$.

The fact that $Z F C$ radically underdetermines cardinal arithmetic is particularly evident in Easton's striking result:

Easton theorem. For regular cardinals $\aleph_{\alpha}$, one can impose via forcing in $Z F C$ the power function $2^{\aleph_{\alpha}}=\aleph_{F(\alpha)}$ for quite every function $F$ satisfying (i) and (ii).

For regular cardinals $\kappa$, we have $\kappa^{\mathrm{cf}(\kappa)}=\kappa^{\kappa}=2^{\kappa}$ but for singular cardinals $\sigma$ we have $\sigma^{\mathrm{cf}(\sigma)}=\left(\begin{array}{c}\sigma \\ \operatorname{cf}(\sigma)\end{array}\right) 2^{\operatorname{cf}(\sigma)}$ where $\left(\begin{array}{c}\kappa \\ \lambda\end{array}\right)$ for $\kappa>\lambda$ is a sophisticated generalization of the binomial formula.

The proof of Easton theorem uses iterated Cohen forcing.

\footnotetext{
${ }^{11}$ See Jech [1978], p. 49.
} 


\subsection{Cohen forcing}

Cohen forcing (1963) 12 allows to construct in a very systematic way "generic" extensions $N$ of inner models $M$ of $Z F$ or $Z F C$ (that is transitive $\in$-submodels $M \subset V$ of $Z F$ or $Z F C$ with $O n \subseteq M)$ where some desired properties become valid.

Suppose for instance that, starting with a ground inner model $M$ of $Z F C$ in $V$, we want to construct another inner model $N$ where $\omega_{1}^{M}$ (that is the cardinal $\omega_{1}=\aleph_{1}$ as defined in $M$ ) collapses and becomes countable. We need to have at our disposal in $N$ a surjection $f: \omega \rightarrow \omega_{1}^{M}$ that, by definition of $\omega_{1}^{M}$, cannot belong to $M$. Suppose nevertheless that such an $f$ exists. Then for every $n$ the restriction $\left.f\right|_{n}=(f(0), \ldots, f(n-1))$ exists and is an element of the ground model $M$. Let us therefore consider the set $P=\{p\}$ of finite sequences $p=\left(\alpha_{0}, \ldots, \alpha_{n-1}\right)$ of countable ordinals $\alpha_{i}<\omega_{1}^{M}$ of $M$. Such $p$ are called forcing conditions and must be interpreted as forcing $\left.f\right|_{n}=p$. The set $P$ exists, is well defined in $M$, and is endowed with a natural partial order " $q \leq p$ iff $p \subseteq q$ " 13 . If $f$ exists, we can consider $G=\left\{\left.f\right|_{n}\right\}_{n \in \mathbb{N}}$ which is a subset of $P$ in $V$ s.t. $\cup G=f$. But as $f \notin M, G$ cannot be a subset of $P$ in $M$.

If $f$ exists, it is trivial to verify that $G$ satisfies the following properties:

1. Gluing and restriction conditions (see topos theory): if $p, q \in G$, then $p$ and $q$ are initial segments of $f$ and are compatible in the sense that $p \leq q$ or $q \leq p$ and therefore there exists a common smaller element $r \in G$ satisfying $r \leq p, r \leq q$.

2. for every $n \in \omega$, there exists $p \in G$ s.t. $n \in \operatorname{dom}(p)$ (i.e. $\operatorname{dom}(f)=\omega)$.

3. for every $M$-countable ordinal $\alpha<\omega_{1}^{M}$, there exists $p \in G$ s.t. $\alpha \in \operatorname{range}(p)$ (i.e. range $(f)=\omega_{1}^{M}$, it is the fundamental condition of surjectivity for the collapsing of $\omega_{1}^{M}$ in $N$ ).

Cohen's idea is to construct sets $G$ in $V$ satisfying these properties and to show that extending the ground inner model $M$ by such a $G$ yields an appropriate inner model $N=M[G]$ which is the smaller inner model of $V$ containing $M$ and $G$.

So, one supposes that a partially ordered set of forcing conditions $P$ is given. A subset of conditions $D \subseteq P$ is called dense if for every

\footnotetext{
${ }^{12}$ Awarded a Fields Medal in 1966, Paul Cohen died on March 23, 2007.

${ }^{13}$ That is $q<p$ means that $q$ forces a better approximation of $f$ than $p$.
} 
$p \in P$ there is a smaller $d \leq p$ belonging to $D$. One then defines generic classes $G \subseteq P$ of conditions. $G \notin M$ is generic over $M$ iff:

(i) $p \in G$ and $p \leq q \in P$ implies $q \in G$,

(ii) for every $p, q \in G$, there exists a common smaller $r \in G$ satisfying $r \leq p, r \leq q$,

(iii) for every dense set $D$ of conditions $D \in M$ (be careful: $D \in M$, $D \subseteq P \in M, G \subseteq P$, but $G \notin M)$, there exists $p \in D$ such that $p \in G$ (i.e. $G \cap D \neq \emptyset$ ).

Properties (i) and (ii) mean that $G$ is a filter for the order $\leq$. If $G$ is generic, the properties (2) and (3) above are automatically satisfied since the sets of conditions $D_{n}=\{p \in P: n \in \operatorname{dom}(p)\}$ for $n \in \omega$ and $E_{\alpha}=\{p \in P: \alpha \in \operatorname{range}(p)\}$ for $\alpha<\omega_{1}^{M}$ are dense: (2) means $G \cap D_{n} \neq \emptyset$ and (3) means $G \cap E_{\alpha} \neq \emptyset$.

Cohen's main theorem. There exists a $Z F C$-model $\mathcal{A}=M[G]$ such that (1) $M$ is an inner model of $\mathcal{A},(2) G$ is not a set in $M$ but is a set in $\mathcal{A},(3)$ if $\mathcal{A}^{\prime}$ is another model statisfying (1) et (2), then there exists an elementary embedding $j: \mathcal{A} \prec \mathcal{A}^{\prime}$ such that $j(\mathcal{A})$ is an inner model of $\mathcal{A}^{\prime}$ and $\left.j\right|_{M}=\operatorname{Id}(M)$, (4) $\mathcal{A}$ is essentially unique.

If $j: \mathcal{A} \prec \mathcal{A}^{\prime}$ is an embedding of a model $\mathcal{A}$ in a model $\mathcal{A}^{\prime}$, elementarity means that $\mathcal{A}^{\prime}$ has exactly the same first-order theory as $\mathcal{A}$ in the language $\mathcal{L}_{\mathcal{A}}$ where there exist names for every element of $\mathcal{A}$ (that is for every set $x$ and first-order formula $\varphi, \mathcal{A} \models \varphi(x)$ iff $\mathcal{A}^{\prime} \models \varphi(j(x))$. So, in the first-order case, $\mathcal{A}^{\prime}$ adds only indiscernible elements. A less constraining relation is elementary equivalence: $\mathcal{A}$ and $\mathcal{A}^{\prime}$ are elementary equivalent, $\mathcal{A} \equiv \mathcal{A}^{\prime}$, if they have the same firstorder theory. In that case, elements of $\mathcal{A}$ characterized by first-order sentences can be substituted for other elements of $\mathcal{A}^{\prime}$. It is no longer the case for an elementary embedding.

An essential feature of forcing extensions is that it is possible to describe $M[G]$ using the language $\mathcal{L}_{G}$ which is the language $\mathcal{L}$ of $M$ extended by a new symbol constant for $G$. As was emphasized by Patrick Dehornoy (2003), forcing is

"as a field extension whose elements are described by polynomials defined on the ground field".

In particular, the validity of a formula $\varphi$ in $M[G]$ can be coded by a forcing relation $p \Vdash \varphi$ defined in $M$. The definition of $p \Vdash \varphi$ is rather technical but an excellent intuition is given by the idea of 
"localizing" truth, $p$ being interpreted as a local domain (as an open set of some topological space), and $p \Vdash \varphi$ meaning that $\varphi$ is "locally true everywhere" on $p$.

Forcing theorem. For every generic $G \subseteq P, M[G] \models \varphi$ iff there exists a $p \in G$ s.t. $p \Vdash \varphi$.

Using forcing, we can add to $\mathbb{R}$ (i.e. to $\mathcal{P}(\omega)$ ) new elements called generic reals. Let $P$ be the partial order of binary finite sequences $p=(p(0), \ldots, p(n-1))$. If $G \subseteq P$ is generic, $f=\cup G$ is a map $f$ : $\omega \rightarrow\{0,1\}$ which is the characteristic function $f=1_{A}$ of a new subset $A \subseteq \omega$ and $A \notin M$. Indeed, if $g: \omega \rightarrow\{0,1\}$ defines a subset $B \subseteq \omega$ which belongs to $M$, then the set of conditions $D_{g}=\{p \in P: p \nsubseteq g\} \in$ $M$ is dense (if $p$ is any finite sequence it can be extended to a sequence long enough to be different from $g$ ) and therefore $G \cap D_{g} \neq \emptyset$. But this means $f \neq g$.

To prove the negation $\neg C H$ of $C H$, one adds to $M$ a great number of generic reals. More precisely, one embeds $\omega_{2}^{M}$ into $\{0,1\}^{\omega}$ (isomorphic to $\mathbb{R}$ ) using as forcing conditions the set $P$ of finite binary sequences of $\omega_{2}^{M} \times \omega$. If $G$ is generic, then $f=\cup G$ is a map $f: \omega_{2}^{M} \times \omega \rightarrow\{0,1\}$, that is an $\omega_{2}^{M}$-family $f=\left\{f_{\alpha}\right\}_{\alpha<\omega_{2}^{M}}$ of generic reals $f_{\alpha}: \omega \rightarrow\{0,1\}$. Using density arguments one shows that $f$ yields an embedding $\omega_{2}^{M} \hookrightarrow\{0,1\}^{\omega}$ in $M[G]$ and that $\omega_{2}^{M}$ doesn't collapse in $M[G]$ (because $P$ is $\omega$-saturated, i.e. there doesn't exist in $P$ any infinite countable subset of incompatible elements). This implies immediately $\neg \mathrm{CH}$.

Easton theorem is proved by iterating such constructions and adding to every regular $\aleph_{\alpha}$ as many new subsets as it is necessary to have $2^{\aleph_{\alpha}}=\aleph_{F(\alpha)}$.

\subsection{Absoluteness}

Many philosophers and logicians which are "deflationist" regarding mathematical "ontology" consider that the only sentences having a well determined truth-value are those the truth-value of which is the same in all models of $Z F C$, and that sentences the truth-value of which can change depending on the chosen model are "inherently vague". Such an antiplatonist conception has drastic consequences. Indeed, contrary to first order arithmetic, which is $Z F$-absolute, that is invariant relative to extensions of the universe (Schönfield theorem), all structures and notions such as $\mathcal{N}, \mathbb{R}, \operatorname{Card}(\chi), x \rightarrow \mathcal{P}(x), x \rightarrow|x|$, and second order arithmetic, are not $Z F$-absolute. They can vary 
widely from one model to another and can't have absolute truth value in $Z F$. This "vagueness" is one of the main classical arguments of antiplatonists against non-constructive set theories. But, it has been emphasized by Hugh Woodin in his 2003 paper Set theory after Russell. The journey back to Eden that vagueness is not an admissible argument against platonism and shows only that it is necessary to classify the different models of $Z F$ and $Z F C$. As he explained also in his talk at the Logic Colloquium held in Paris in 2000 (quotation from Dehornoy, 2003, p. 23):

"There is a tendency to claim that the Continuum Hypothesis is inherently vague and that this is simply the end of the story. But any legitimate claim that $\mathrm{CH}$ is inherently vague must have a mathematical basis, at the very least a theorem or a collection of theorems. My own view is that the independence of $C H$ from $Z F C$, and from $Z F C$ together with large cardinal axioms, does not provide this basis. (...) Instead, for me, the independence results for $\mathrm{CH}$ simply show that $\mathrm{CH}$ is a difficult problem."

In fact, the strong variability of the possible models of $Z F C$ is an argument in favor of the irreducibility of the continuum to a set of points which can be "individuated" by a symbolic description.

To tackle this problem, we must look at two opposed strategies, both introduced by Gödel, one being "minimalist" ("ontologically" deflationist) and the other "maximalist" ("ontologically" inflationist), and first introduce some classes of sets of reals.

\section{Borel and projective hierarchies}

In descriptive set theory, one works in $\mathbb{R}$ or in $\mathcal{N}=\omega^{\omega}$ or in $\{0,1\}^{\omega}$, and, more generally, on metric, separable, complete, perfect (closed without isolated points) spaces $\mathcal{X}$ (Polish spaces). One considers in $\mathcal{X}$ different "nicely" definable classes of subsets $\Gamma$. The first is the Borel hierarchy constructed from the open sets by iterating the operations of complementation and of "projection" $\mathcal{X} \times \omega \rightarrow \mathcal{X}$. If $P \subseteq \mathcal{X} \times \omega$ (that is, if $P$ is a countable family of subsets $P_{n} \subseteq \mathcal{X}$ ), one considers the subset of $\mathcal{X}$ defined by $\exists^{\omega} P=\{x \in \mathcal{X} \mid \exists n P(x, n)\}$ It it is the union $\bigcup_{n \in \omega} P_{n}$.

\footnotetext{
${ }^{14}$ We identify predicates $\varphi(x), P(x, n)$, etc. with their extensions.
} 
The $\Sigma_{1}^{0}$ are the open subsets of $\mathcal{X}$, the $\Pi_{1}^{0}=\neg \Sigma_{1}^{0}$ are the closed subsets, $\Delta_{1}^{0}=\Pi_{1}^{0} \cap \Sigma_{1}^{0}$ the clopen subsets, and the Borel hierarchy $B$ is defined by:

$\Pi_{n}^{0}=\left\{\neg \varphi \mid \varphi \in \Sigma_{n}^{0}\right\}=\neg \Sigma_{n}^{0}, \Sigma_{n+1}^{0}=\exists^{\omega} \neg \Sigma_{n}^{0}=\exists^{\omega} \Pi_{n}^{0}, \Delta_{n}^{0}=\Pi_{n}^{0} \cap \Sigma_{n}^{0}$.

It can be shown that this hierarchy is strict:

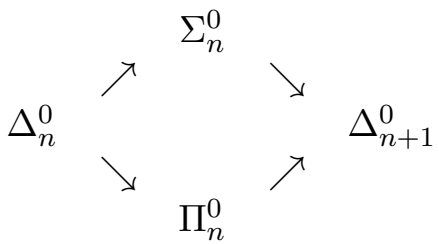

One then defines the higher hierarchy of projective sets using a supplementary principle of construction, namely projections by continuous projections $\mathcal{X} \times \mathcal{N} \rightarrow \mathcal{X}$, written $\exists^{\mathcal{N}}$. One gets a new hierarchy beginning with the class $\Sigma_{1}^{1}=\exists^{\mathcal{N}} \Pi_{1}^{0}$ - the so called analytic subsets and continuing with the classes:

$\Pi_{n}^{1}=\left\{\neg \varphi \mid \varphi \in \Sigma_{n}^{1}\right\}=\neg \Sigma_{n}^{1}, \Sigma_{n+1}^{1}=\exists^{\mathcal{N}} \neg \Sigma_{n}^{1}=\exists^{\mathcal{N}} \Pi_{n}^{1}, \Delta_{n}^{1}=\Pi_{n}^{1} \cap \Sigma_{n}^{1}$.

For instance, $P \subseteq \mathcal{X}$ is $\Sigma_{1}^{1}$ if there exists a closed subset $F \subseteq \mathcal{X} \times \mathcal{N}$ such that: $P(x) \Leftrightarrow \exists \alpha F(x, \alpha)$. In the same way, $P \subseteq \mathcal{X}$ is $\Sigma_{2}^{1}$ if there exists an open subset $G \subseteq \mathcal{X} \times \mathcal{N} \times \mathcal{N}$ such that: $P(x) \Leftrightarrow$ $\exists \alpha \forall \beta G(x, \alpha, \beta)$, etc.

More generally, one can define projective sets in $V$ using the cumulative hierarchy of successive levels of $V$ indexed by the class $O n$ of ordinals: $V_{0}=\emptyset, V_{\alpha+1}=\left\{x: x \subset V_{\alpha}\right\}$ for a successor ordinal, and $V_{\lambda}=\bigcup_{\alpha<\lambda} V_{\alpha}$ for $\lambda$ a limit ordinal. Then $P$ is projective if it is definable with parameters over $\left(V_{\omega+1}, \in\right)$. More precisely, $P \subset V_{\omega+1}$ is $\Sigma_{n}^{1}$ if it is the set of sets $x$ s.t. $\left(V_{\omega+1}, \in\right) \models \varphi(x)$ for a $\Sigma_{n}$ formula $\varphi(x)$, that is a formula of the form $\varphi(x)=\exists x_{1} \forall x_{2} \ldots \psi$ with $n$ quantifiers and a $\psi$ having only bounded quantifiers 15

As the Borel hierarchy, the projective hierarchy is strict and it is a continuation of the Borel hierarchy according to:

Suslin theorem. $B=\Delta_{1}^{1}$.

\footnotetext{
${ }^{15}$ Bounded quantifiers are of the form $\exists y \in z$ and $\forall y \in z$.
} 
This theorem can be interpreted as a construction principle: it asserts that the complex operation of continuous projection can be reduced to an iteration of simpler operations of union and complementation.

There exist strict $\Pi_{n}^{1}$ and $\Sigma_{n}^{1}$ sets, which are very natural in classical analysis. For instance, in the functional space $C[0,1]$ of real continuous functions on $[0,1]$ endowed with the topology of uniform convergence, the subset

$$
\{f \in C[0,1] \mid f \operatorname{smooth}\}
$$

is $\Pi_{1}^{1}$ (but not $\Delta_{1}^{1}$ ). In the space $C[0,1]^{\omega}$ of countable sequences $\left(f_{i}\right)$ of functions, the subset:

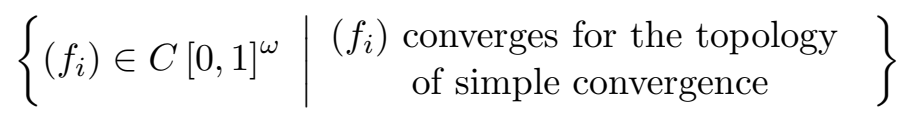

is $\Pi_{1}^{1}$, and the subset:

$$
\left\{\begin{array}{l|l}
\left(f_{i}\right) \in C[0,1]^{\omega} & \begin{array}{l}
\text { a sub-sequence converges for the } \\
\text { topology of simple convergence }
\end{array}
\end{array}\right\}
$$

is $\Sigma_{2}^{1}$ and every $\Sigma_{2}^{1}$ can be represented that way (Becker [1992]):

Becker representation theorem. For every $\Sigma_{2}^{1}$-set $S \subseteq C[0,1]$ there exists a sequence $\left(f_{i}\right)$ such that

$$
S=A_{\left(f_{i}\right)}=\left\{g \in C[0,1] \mid \begin{array}{c}
\text { a sub-sequence of }\left(f_{i}\right) \text { converges } \\
\text { towards } g \text { for the topology } \\
\text { of simple convergence }
\end{array}\right\} .
$$

Another examples are given by the compact subsets $K \in \mathcal{K}\left(\mathbb{R}^{n}\right)$ of $\mathbb{R}^{n}$ : for $n \geq 3$,

$$
\left\{K \in \mathcal{K}\left(\mathbb{R}^{n}\right) \mid K \text { arc connected }\right\}
$$

is $\Pi_{2}^{1}$, and for $n \geq 4$,

$$
\left\{K \in \mathcal{K}\left(\mathbb{R}^{n}\right) \mid K \text { simply connected }\right\}
$$

is also $\Pi_{2}^{1}$.

In fact, projective sets can be considered as the "reasonably" definable subsets of $\mathbb{R}$. 


\section{The "minimalist" strategy of the con- structible universe}

The first Gödelian strategy for constraining the structure of $Z F$ universes consisted in restricting the universe $V$. It is the strategy refered to as $V=L$ - of constructible sets (Gödel 1938).

To define $L$ one substitutes, in the construction of the cumulative hierarchy $V_{\alpha}$ of $V$ by means of a transfinite recursion on the $x \rightarrow \mathcal{P}(x)$ operation, the power sets $\mathcal{P}(x)$ - which are not $Z F$ absolute - with smaller sets $\mathcal{D}(x)=\{y \subseteq x \mid y$ elementary $\}$ (where "elementary" means definable by a first order formula over the structure $\langle x, \in,\{s \mid s \in x\}\rangle)$ - which are $Z F$-absolute. $L$ is then defined as $V$ using a transfinite recursion on ordinals: $L_{0}=\emptyset, L_{\alpha+1}=\mathcal{D}\left(L_{\alpha}\right)$, $L_{\lambda}=\bigcup_{\alpha<\lambda} L_{\alpha}$ if $\lambda$ is a limit ordinal, and $L=\bigcup_{\alpha \in O n} L_{\alpha}$. The absoluteness of $L$ comes from the fact that each level $L_{\alpha}$ is constructed using only unambiguous formulae and parameters belonging to the previous stages $L_{\beta}, \beta<\alpha$.

Gödel $(1938,1940)$ has shown that if $V=L$ it is possible to define a global wellordering on $L$, which is a very strong form of global $A C$. The wellorder relation is defined by a transfinite induction on the levels $\alpha$. If $x$ and $y$ are of different levels their order is the order of their respective levels. If they are of the same level, their order is first that of the Gödel numbers of their minimal defining formulae, and then the order of their parameters (which are of lower order and therefore wellordered by the induction hypothesis). Gödel also proved that in $Z F$ we have $(V=L) \vdash G C H$.

$L$ is in fact the smallest inner model of $V$ :

(i) $O n \subset L$,

(ii) $L$ is transitive: if $y \in_{V} x$ and $x \in_{L} L$, then $y \in_{L} L$,

(iii) $\left(L, \in_{L}\right)$ is a model of $Z F$.

It can be defined in $V$ by a statement $L(x)=$ " $x$ is constructible" which is independent of $V$ ( $Z F$-absolute), and in that sense, it is a canonical model of $Z F C$.

Remark. It must be emphasized that the constructible universe $L$ is not constructive since it contains the class $O n$ of ordinals which is non constructive. But the characteristic property of $L$ is that it reduces non-constructivity exactly to $\mathrm{On}$. 
In the constructible universe $L$ there exists a $\Delta_{2}^{1}$-wellorder relation $<$ on $\mathbb{R}$. According to a theorem due to Fubini, such a wellordering cannot be Lebesgue measurable and there exist therefore in $L \Delta_{2}^{1}$ sets which, despite the fact they belong to the low levels of the projective hierarchy and are "simple" and "nice" to define, are nevertheless not Lebesgue measurable and therefore not well-behaved.

With regards to $C H$, one uses the fact that the $\Delta_{2}^{1}$-wellorder relation $<$ on $\mathbb{R}$ is a fortiori $\Sigma_{2}^{1}$, and that the $\Sigma_{2}^{1}$ are the $\aleph_{1}$-Suslin sets. If $\chi$ is an infinite cardinal, $P \subseteq \mathbb{R}$ is called a $\chi$-Suslin set if it exists a closed subset $F \subseteq \mathbb{R} \times \chi^{\mathbb{N}}$ s.t. $P=\exists \chi^{\mathbb{N}} F$ (i.e. $P$ is the projection of $F)$. The $\Sigma_{1}^{1}$ are, by definition, the $\aleph_{0}$-Suslin sets. Indeed, if $\chi=\aleph_{0}$ then $P=\exists^{\mathbb{R}} F$ and therefore $P \in \Sigma_{1}^{1}$. A theorem of Martin says that $P \subseteq \mathcal{X}$ is an $\aleph_{n}$-Suslin set iff $P=\bigcup_{\xi<\aleph_{n}} P_{\xi}$ with $P_{\xi}$ Borelians 16 As the wellordering $<$ on $\mathbb{R}$ is $\Sigma_{2}^{1}$, according to a theorem of Schönfield, its ordinal is $<\aleph_{2}$ and $C H$ is therefore valid.

In spite of its intrinsic limitations, $L$ is a very interesting model of $Z F C$, which possesses a "fine structure" interpolating between the different $F_{\alpha}$ and very rich combinatorial properties investigated by Jensen. One of its main properties is the following. Let us first define what is a club ("closed unbounded" subset) $C \subseteq \alpha$ of a limit ordinal $\alpha$ : $C$ is closed for the order topology (i.e. limits in $C$ belong to $C$ : if $\beta<\alpha$ and $\operatorname{Sup}(C \cap \beta)=\beta$, then $\beta \in C)$ and unbounded in $\alpha$ (for every $\beta<\alpha$ ) there exists an element $\gamma \in C$ s.t. $\beta<\gamma$. For a cardinal $\kappa$, let $\square_{\kappa}$ be the property that there exists a sequence of clubs $C_{\alpha} \subseteq \alpha$ with $\alpha<\kappa^{+}$s.t. $C_{\alpha}$ is of order type $\leq \kappa$ (and $<\kappa$ if $\operatorname{cf}(\alpha)<\kappa)$ and if $\lambda$ is a limit point of $C_{\alpha}$ then $C_{\alpha} \cap \lambda=C_{\lambda} . \square_{\kappa}$ is used to construct systematically and coherently bijections between $\kappa$ and ordinals $\kappa \leq \alpha<\kappa^{+}$by cofinalizing the $\alpha$ by clubs. We have:

Theorem (Jensen, 1970). $V=L \models \forall \kappa \square_{\kappa}$.

$\square_{\kappa}$ constrains the structure of the stationary subsets $S$ of $\kappa^{+}(S \subset$ $\kappa^{+}$is stationary if $S \cap C \neq \emptyset$ for every club $C$ in $\left.\kappa^{+}\right)$. These cannot reflect at some ordinal $\alpha<\kappa$ of $\operatorname{cf}(\alpha)>\omega$, where "reflect" means "remaining stationary in $\alpha$ ".

One can generalize the concept of constructibility in two ways 17 First, if $A$ is any set, one can relativize definability to $A$ taking $\mathcal{D}_{A}(x)=\{y \subseteq x \mid y$ definable by a first order formula of the structure $\langle x, \in,\{s \mid s \in A \cap x\}\rangle)\}$. One gets that way the universe, called $L[A]$,

\footnotetext{
${ }^{16}$ See Moschovakis [1980], p. 97.

${ }^{17}$ See Kanamori [1994], p. 34.
} 
of constructible sets relative to $A$. In $L[A]$ the only remaining part of $A$ is $A \cap L[A] \in L[A]$. As $L, L[A]$ satisfies $A C$ and is $Z F$-absolute. On the other hand, one can start the recursive construction of $L$ not with $L_{0}=\emptyset$ but with the transitive closure of $\{A\}, L_{0}(A)$. One gets that way $L(A)$ which is the smallest inner model containing $O n$ and $A$. If there is a wellordering on $A$ (it the case if $A C$ is valid), then $L(A)$ is globally wellordered for the same reasons as $L$. In particular, $L(\mathbb{R})$ is a good compromise between the non constructibility of $\mathbb{R}$ and the constructibility from $\mathbb{R}$ of the rest of the universe.

In spite of its interest, the structure of $L$ is rather pathological with regards to the continuum and many of the above results are in some sense counterintuitive. They result from the fact that the $A C$, which implies the existence of very complicated and irregular sets, remains valid in $L$ and that the axiom of constructibility $V=L$ forces some of them to exist inside the projective hierarchy which should be composed only of relatively simple and regular sets: nicely definable sets are not necessarily well-behaved.

It is the reason why many specialists consider that the strategy $V=L$ is dramatically too restrictive and, moreover, that its restriction to constructibility is not philosophically justifiable. For instance, John Steel (2000) claims:

"The central idea of descriptive set theory is that definable sets of reals are free from the pathologies one gets from a wellorder of the reals. Since $V=L$ implies there is a $\Delta_{2}^{1}$ wellorder of the reals, under $V=L$ this central idea collapses low in the projective hierarchy, and after that there is, in an important sense, no descriptive set theory. One has instead infinitary combinatorics on $\aleph_{1}$. This is certainly not the sort of theory that looks useful to Analysts."

One could think that generalizations of constructibility such as $L(A)$ or $L[A]$ would overcome the problem. But it is not the case.

\section{The "maximalist" strategy of large cardinals}

It is therefore justified to reverse the strategy and to look for additional axioms, which could be considered "natural", for $Z F$ and $Z F C$, and to try to generalize to such augmented axiomatics the search of canonical 
models and fine combinatorial structures. As was emphasized by John Steel (2004):

"In extending $Z F C$, we are attempting to maximize interpretative power".

And there is place for philosophy in such a maximizing strategy since the problem is not only to find a solution to the continuum problem but also to understand what "to be a solution" means. By the way, to study such "maximizing" large cardinals models is perfectly compatible with a minimalist perspective: one has only to relativize the theory to the constructible subuniverse $L$ since $Z F C+$ " $V=L " \vdash \varphi$ is equivalent to $Z F C \vdash \varphi^{L}$. As explained by Steel (2004), suppose that the philosopher $A$ believes in $L$ and the philosopher $B$ in $L[G]$ with $G$ forcing the adjunction of $\omega_{2}$ reals to the model of $\mathbb{R}$ in $L$. $A$ believes in $C H$ and $B$ in $\neg C H$, but $B$ can interpret the formulae $\varphi$ of $A$ as its own $\varphi^{L}$ and $A$ can interpret the formulae $\varphi$ of $B$ as forced $\varphi$ (the truth of $\Vdash \varphi$ being definable in the ground model $L$, see above). There is therefore no real conflict.

Different "maximizing" strategies have been considered:

1. Iterate transfinitely theories $T_{\alpha+1}=T_{\alpha}+$ "consistency of $T_{\alpha}$ " starting from $Z F$ or $Z F C$.

2. Postulate "good" regularity properties of projective sets, and therefore of the continuum.

3. Make the theory of the continuum "rigid", that is define under which conditions the properties of $\mathbb{R}$ cannot be further modified by forcing.

Strategy (3) tries to reduce - and even to neutralize - the variability induced by forcing. The ideal aim would be forcing invariance to make the theories of $\mathbb{R}$ and $\mathcal{P}(\mathbb{R})$ in some sense as "rigid" as first order arithmetic. It is an extremely difficult program and we will first evoke some classical results concerning $\mathbb{R}$. CH concerns $\mathcal{P}(\mathbb{R})$ the forcing invariance of which is the object of more recent works of Woodin. But we first emphasize the fact that strategies (1), (2) converge towards the introduction of large cardinal axioms ( $L C A s$ ) which express the existence of higher infinities. Indeed, it seems that every "maximizing" strategy is in some sense equivalent to a $L C A$. Look for instance at the Proper Forcing Axiom PFA. A forcing $P$ is called proper if, for every regular uncountable cardinal $\lambda$, it preserves the stationary subsets of $[\lambda]^{\omega}$ (the set of countable subsets of $\lambda$ ). 
Proper Forcing Axiom. If the forcing $P$ is proper and if the $D_{\alpha}$ 's are dense subsets indexed by the countable ordinals $\alpha<\omega_{1}$, then there exists a filter $G \subseteq P$ intersecting all the $D_{\alpha}$ 's. (Compare with the definition of $G$ being generic).

Many results are known for $P F A$. It implies $2^{\aleph_{0}}=\aleph_{2}$ (and therefore $\neg C H$ ), it implies projective determinacy $P D$ (Woodin) and $A D$ (Steel, 2007) for the inner model $L(\mathbb{R}) 18$ As far as its consistency strength is concerned, it is known that

$$
\operatorname{Con}(\exists \kappa \text { supercompact }) \Rightarrow \operatorname{Con}(P F A) \Rightarrow \operatorname{Con}(\exists \kappa \text { measurable }) \text {. }
$$

It is conjectured that in fact $P F A$ is equiconsistant with " $\exists \kappa$ supercompact" (see below for a definition of supercompacity).

It also seems that there exists a wellordering of $L C A s$ which can be defined by the inclusion of their sets of $\Sigma_{2}^{1}$ (and even $\Pi_{1}^{0}$ ) consequences. As emphasized by John Steel (2000):

"It seems that the consistency strengths of all natural extensions of $Z F C$ are wellordered, and the large cardinal hierarchy provides a sort of yardstick which enables us to compare these consistency strengths."

Philosophically speaking, the nominalist confusion between a strong "quasi-ontology" for sets and a realist "true" ontology of abstract idealities has disqualified such axioms. But I think that such a dogmatic prejudice has been a great philosophical mistake. Indeed, I think that one of the best philosophical formulation of incompleteness is precisely to say that a "good" theory of the continuum requires a very strong "quasi-ontology" for sets, a maximal one, not a minimal one. A "good" regularity of the continuum entails for objective reasons a strong "platonist" commitment concerning higher infinities. This key point has been perfectly emphasized by Patrick Dehornoy:

"properties which put into play objects as 'small' as sets of reals $(. .$.$) are related to other properties which put into$ play very 'huge' objects which seem very far from them.' 19

Some specialists call "reverse descriptive set theory" this remarkable equivalence between properties of regularity of projective sets and LCAs.

\footnotetext{
${ }^{18}$ See below $\S \S 7.2$ and 8 for a definition of $P D$ and $A D$.

${ }^{19}$ Dehornoy [1989].
} 
There are many theorems showing that the platonist "cost" of a "good" theory is very high. Let us for instance mention one of the first striking theorems proved by Robert Solovay using forcing. Let $C M$ be the axiom of existence of a measurable cardinal (see below for a definition).

Solovay theorem (1969). $Z F C+C M \vdash$ every $\Sigma_{2}^{1}$ is "regular" (where "regular" means properties such as Baire property, Lebesgue measurability, and perfect set property) 20

\section{Regularity of projective sets}

\subsection{The regularity of analytic sets}

The French school (Borel, Baire, Lebesgue) and the Russian and Polish schools (Suslin, Luzin, Sierpinski) initiated the study of the Borel and projective classes and achieved deep results concerning their regularity and their representation where "regularity" means Lebesgue measurability, or the perfect set property (to be countable or to contain a perfect subset, i.e. a closed subset without isolated point), or the Baire property (to be approximated by an open subset up to a meager set, i.e. a countable union of nowhere dense sets).

The first regularity theorem is the celebrated:

Cantor-Bendixson theorem. If $A \subseteq \mathbb{R}$ is closed, then $A$ can be decomposed in a unique way as a disjoint union $A=P+S$ where $P$ is perfect and $S$ countable.

As a perfect set $P$ is of cardinality $|P|=2^{\aleph_{0}}$, the continuum hypothesis $C H$ holds for the closed sets $\Pi_{1}^{0}$.

Another early great classical theorem of regularity is the:

Suslin theorem. The analytic subsets $\Sigma_{1}^{1}$ shares the perfect subset property and $\mathrm{CH}$ is therefore true for the $\Sigma_{1}^{1}$ sets.

In the same way, one can show that the $\Sigma_{1}^{1}$ share the Baire property and that the $\Sigma_{1}^{1}$ and $\Pi_{1}^{1}$ are Lebesgue measurable. But it is impossible to show in $Z F$ that the $\Delta_{1}^{1}$ and $\Sigma_{2}^{1}$ share the perfect set property and to show in $Z F C$ that the $\Delta_{2}^{1}$ share the Baire property. In fact many of the "natural" properties of the projective sets go far beyond the demonstrative strength of $Z F$ and $Z F C$. It is therefore methodologically and philosophically justified to look for additional axioms.

\footnotetext{
${ }^{20}$ See Moschovakis [1980], p. 284.
} 


\subsection{Projective determinacy and the "regular- ity" of the continuum}

A very interesting regularity hypothesis is the so called determinacy property. One considers infinite games on sets $X$. Each player (I and II) plays in turn an element $a$ of $X$ :

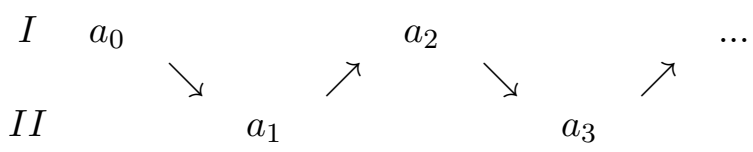

At the end of the game we get a sequence $f \in X^{\mathbb{N}}$. Let $A \subset X^{\mathbb{N}}$. The player I (resp. II) wins the play $f$ of the game $G=G_{X}(A)$ associated to $A$ if $f \in A$ (resp. if $f \notin A$ ).

Definition. $A$ is called determined (written $\operatorname{Det}(A)$ or $\operatorname{Det} G_{X}(A)$ ) if one player has a winning strategy. Therefore $A$ is determined iff

$$
\exists a_{0} \forall a_{1} \exists a_{2} \ldots\left(a_{0}, a_{1}, a_{2}, \ldots\right) \in A \text {. }
$$

Determinacy is a strong property of "regularity". Indeed, for every $A \subset \mathbb{R}$ ( $\mathbb{R}$ being identified with $\left.\mathcal{N}=\omega^{\omega}\right), \operatorname{Det}(A) \Rightarrow " A$ satisfies the Baire and the perfect subset properties, and is Lebesgue measurable".

The first theorem linking determinacy with the projective hierarchy has been the key result 21

Gale-Stewart Theorem(1953). $Z F C \vdash$ closed subsets $A$ of $X^{\mathbb{N}}$ (the $\left.\Pi_{1}^{0}\right)$ are determined.

After many efforts, Donald Martin proved a fundamental theorem which concluded a first stage of the story:

Martin theorem (1975). $Z F C \vdash$ Borel sets (the $\Delta_{1}^{1}$ ) are determined.

This celebrated result shows that $Z F C$ is a "good" axiomatic for the Borel subsets of $\mathbb{R}$. But, it is the limit of what is provable in $Z F C$. Indeed, $Z F C$ cannot imply the determinacy of $\Sigma_{1}^{1}$-sets since in the constructible model $L$ of $Z F C$ there exist $\Sigma_{1}^{1}$-sets that don't share the perfect set property. As for $\Pi_{1}^{1}$-sets, their determinacy implies the measurability of the $\Sigma_{2}^{1}$-sets, but in $L$ there exists a $\Delta_{2}^{1}$-wellorder of $\mathbb{R}$, which, according to Fubini theorem, cannot be Lebesgue measurable.

\footnotetext{
${ }^{21}$ See Grigorieff [1976] and Moschovakis [1980], p. 288.
} 


\section{The necessity of large cardinals and "reverse" descriptive set theory}

To prove determinacy results for projective sets beyond $\Delta_{1}^{1}$, one must introduce additional axioms and many converging results show that the most natural are large cardinal axioms. The first example was introduced by Stan Ulam. If $X$ is a set, a filter $\mathcal{U}$ over $X$ is a set of subsets of $X, \mathcal{U} \subseteq \mathcal{P}(X)$, s.t. (i) $\emptyset \notin \mathcal{U}$, (ii) if $U \in \mathcal{U}$ and $U \subseteq V$ then $V \in \mathcal{U}$, (iii) if $U, V \in \mathcal{U}$ then $U \cap V \in \mathcal{U}$ (i.e., the complementary set of $\mathcal{U}$ in the Boolean algebra $\mathcal{P}(X)$ is an ideal). $\mathcal{U}$ is an ultrafilter if it is maximal, namely if for every $U \subseteq X$, either $U \in \mathcal{U}$ or $X-U \in$ $\mathcal{U}$. For every $x \in X, \mathcal{U}_{x}=\{U \subseteq X: x \in U\}$ is an ultrafilter called "principal". A non principal ultrafilter is called "free".

Definition. A cardinal $\chi>\omega$ is measurable if it bears a free ultrafilter $\mathcal{U}$ which is $\chi$-complete (that is stable w.r.t. $\chi$-infinite intersections $\bigcap_{\lambda<\chi} X_{\lambda}$ with $\left.\lambda<\chi\right)$. It is equivalent to say that $\chi$ bears a measure $\mu$ with range $\{0,1\}$ (with $\mu(\chi)=1$ ), diffuse (without atoms: $\forall \xi \in \chi$ we have $\mu(\{\xi\})=0)$ and $\chi$-additive. The equivalence is given by $\mu(A)=1 \Leftrightarrow A \in \mathcal{U}$ and $\mu(A)=0 \Leftrightarrow \chi-A \in \mathcal{U}$ and is analogous to the opposition between finite and infinite subsets in $\omega$.

A first typical result was another theorem due to Donald Martin:

Martin theorem (1970). $Z F C+M C \vdash \operatorname{Det}\left(\Sigma_{1}^{1}\right)$.

Corollary: Solovay theorem (1969). $Z F C+M C \vdash$ "the $\Sigma_{2^{-}}^{1}$ sets are 'regular'".

But Solovay also showed that $Z F C+M C \nvdash P D$ (where $P D$ is the axiom of Projective Determinacy: every projective $A \subseteq \mathbb{R}$ is determined, see below) since $Z F C+P D \vdash \operatorname{Cons}(Z F C+M C)$ and therefore if $Z F C+M C \vdash P D$ we would have $Z F C+M C \vdash \operatorname{Cons}(Z F C+M C)$, which would contradict Gödel theorem.

Scott theorem (1961). $M C$ is false in $V=L$ and therefore $Z F C \nvdash C M$.

The proof of Scott theorem uses the concept of an ultrapower $V^{\mathcal{U}}$ where $\mathcal{U}$ is an ultrafilter on a set $S$. The elements of $V^{\mathcal{U}}$ are the maps $f: S \rightarrow V, f$ and $g$ being equivalent if they are equal almost everywhere (a.e.), that is if $\{s \in S: f(s)=g(s)\} \in \mathcal{U}$. Any element $x$ of $V$ is represented by the constant map $f(s)=x$ and this defines a canonical embedding $j: V \hookrightarrow V^{\mathcal{U}}$. If $\varphi\left(x_{1}, \ldots, x_{n}\right)$ is a formula of the language of $V, \varphi\left(f_{1}, \ldots, f_{n}\right)$ is valid in $V^{\mathcal{U}}\left(V^{\mathcal{U}} \models \varphi\left(f_{1}, \ldots, f_{n}\right)\right)$ iff 
$\varphi\left(f_{1}, \ldots, f_{n}\right)$ is valid a.e., that is if

$$
\left\{s \in S: V \models \varphi\left(f_{1}(s), \ldots, f_{n}(s)\right)\right\} \in \mathcal{U} .
$$

One shows that $V^{\mathcal{U}}$ is well-founded if the ultrafilter $\mathcal{U}$ is $\omega_{1}$-complete and that there exists an isomorphism between $\left\langle V^{\mathcal{U}}, \in \mathcal{U}\right\rangle$ and $\left\langle M_{\mathcal{U}}, \in\right\rangle$ where $M_{\mathcal{U}}$ is an inner model (Mostowski collapsing lemma). A fundamental theorem of Łoš says that $j: V \prec M_{\mathcal{U}}$ is an elementary embedding (see above for the definition). If $j$ is an elementary embedding $j: M \prec M^{*}$ of models of $Z F C$ where $M^{*}$ is an inner model of $M$ and if $\alpha \in O n(M)$ is an ordinal in $M$, one has $j(\alpha) \in O n\left(M^{*}\right) \subset O n(M)$ and, because of the elementarity of $j, \alpha<\beta \Leftrightarrow j(\alpha)<j(\beta)$. This implies $j(\alpha) \geq \alpha$. One shows that there exists necessarily an ordinal $\alpha$ s.t. $j(\alpha)>\alpha$. Let $\chi$ be the smallest of these $\alpha$. It is called the critical ordinal crit $(j)$ of $j$.

Theorem. If the free ultrafilter $\mathcal{U}$ on the measurable cardinal $\chi$ is $\chi$-complete, then $\operatorname{crit}(j)=\chi$ and therefore $j(\chi)>\chi$.

\section{Corollary: Scott theorem.}

Indeed, suppose there exists a $M C$ and let $\chi$ be the least $M C$. Now suppose that $V=L$. Elementarity implies $M_{\mathcal{U}}=L$ since $M_{\mathcal{U}}$ is an inner model satisfying the axiom of constructibility and therefore $L \subseteq M_{\mathcal{U}} \subseteq V=L$. Then in $M_{\mathcal{U}}=L=V, j(\chi)$ is the least $M C$, which contradicts $j(\chi)>\chi$.

Measurable cardinals $\chi$ are very large; such a $\chi$ is regular (there exists no unbounded $f: \lambda \rightarrow \chi$ with $\lambda<\chi$ ), strongly inaccessible $\left(\forall \lambda<\chi, 2^{\lambda}<\chi\right)$, and preceded by $\chi$ strongly inaccessible smaller cardinals. But as large as they may be, $M C s$ guarantee only the determinacy of the lowest post-Borelian level of definable subsets of $\mathbb{R}$. To guarantee the determinacy of all projective subsets, one needs much stronger axioms, such as $P D$, which are not entailed by $M C$ (see Solovay's remark above)

As we will see below, many specialists consider that Projective Determinacy is a "good" axiomatic for $\mathbb{R}$. Indeed, $P D$ is "empirically complete" for the projective sets and $Z F C+P D$ "rigidifies" the properties of projective sets w.r.t. forcing: it makes them "forcingabsolute" or "generically absolute". One can also consider the even stronger axiom (Woodin axiom) " $L(\mathbb{R})$ satisfies $A D$ " where $L(\mathbb{R})$ is the constructible closure of $\mathbb{R}$ (i.e. the smallest inner model containing $O n$ and $\mathbb{R}$, see above) and the Axiom of Determinacy $A D$ means that every subset of $\mathbb{R}$ is determined. $A D$ is incompatible with $A C$ since, 
according to Fubini theorem, $A C$ enables to construct non-Lebesgue measurable, and therefore non-determined, subsets of $\mathbb{R}$.

\section{The transcendance of $\mathbb{R}$ over $L$ and the set $0^{\#}$ (0 sharp)}

\subsection{Indiscernible ordinals}

Once we accept the relevance and the legitimacy of $L C A s$, we need some tools for measuring the transcendence of $V$ over $L$. A first possibility is given by what are called indiscernible ordinals (Silver, 1966) which enable to construct the simplest canonical non constructible real. We consider the levels of $L$ of the form $\left\langle L_{\lambda}, \in\right\rangle$ with $\lambda$ a limit ordinal. A set $I$ of ordinals in this cumulative hierarchy $L_{\lambda}$ of constructive sets up to level $\lambda$ is called a set of indiscernibles if, for every $n$-ary formula $\varphi\left(x_{1}, \ldots, x_{n}\right)$, the validity of $\varphi$ on $I$ is independent of the choice of the $x_{i}$ 's: that is for every sequences $c_{1}<\ldots<c_{n}$ and $d_{1}<\ldots<d_{n}$ in $I$

$$
L_{\lambda} \models \varphi\left(c_{1}, \ldots, c_{n}\right) \text { iff } L_{\lambda} \models \varphi\left(d_{1}, \ldots, d_{n}\right) .
$$

When it exists, the set $S$ of Silver indiscernibles is characterized by the following properties, which express that, for all uncountable cardinals $\kappa$, all the $L_{\kappa}$ 's share essentially the same first-order structure:

1. $\kappa \in S$ (all uncountable cardinals of $V$ are indiscernible in $L$ ).

2. $S \cap \kappa$ is of order-type $\kappa$.

3. $S \cap \kappa$ is closed and unbounded (club, see above) in $\kappa$ if $\kappa$ is regular.

4. $S \cap \kappa$ is a set of indiscernibles for $\left\langle L_{\kappa}, \in\right\rangle$.

5. The Skolem hull of $S \cap \kappa$ in $L_{\kappa}$ is equal to $L_{\kappa}$ : $\operatorname{Hull}^{L_{\kappa}}(S \cap \kappa)=$ $L_{\kappa}$, where the Skolem hull of $I \subset L_{\kappa}$ is constructed by adding for every $(n+1)$-ary formula $\varphi\left(y, x_{1}, \ldots, x_{n}\right)$ with $x_{i} \in I$ a Skolem term $t_{\varphi}\left(x_{1}, \ldots, x_{n}\right)$ which is the smallest $y$ (for the wellorder of $L)$ s.t. $\varphi\left(y, x_{1}, \ldots, x_{n}\right)$ if such an $y$ exists and 0 otherwise. In other words every constructible element $a \in L_{\kappa}$ is definable by a definite description with parameters in the indiscernibles $S \cap \kappa$. 
This can be generalized to structures $\mathcal{M}=\langle M, E\rangle$ with a binary relation $E$ looking like $\in$, that is, which are elementary equivalent to some $\left\langle L_{\lambda}, \in\right\rangle$ for $\lambda$ a limit ordinal and $I \subset M$. In that case, we have $\operatorname{Hull}^{\mathcal{M}}(I) \prec \mathcal{M}$ and in fact $\operatorname{Hull}^{\mathcal{M}}(I)$ is the smallest elementary substructure of $\mathcal{M}$ containing $I$. Let $\Sigma=\Sigma(\mathcal{M}, I)$ be the set of formulae $\varphi$ which can be satisfied by $\mathcal{M}$ on $I$. This defines particular sets of formulae called $E M$-sets (from Ehrenfeucht-Mostowski, 1956). The EM theorem says that if $\Sigma$ is a theory having infinite models and if $\langle I,<\rangle$ is any total well-ordering of infinite order-type $\alpha \geq \omega$, then there exists a model $\mathcal{M}$ of $\Sigma$ containing $I$ for which $I$ is a set of indiscernibles, and moreover, $\mathcal{M}$ can be chosen in such a way as to be the Skolem hull of $I: \mathcal{M}=\operatorname{Hull}^{\mathcal{M}}(I)$. Such an $(\mathcal{M}, I)$ is essentially unique and its transitive collapse (isomorphism with a structure where $E$ becomes $\in)$ is written $(\mathcal{M}(\Sigma, \alpha), I(\Sigma, \alpha))$ where $\alpha$ is the order type of $I(I(\Sigma, \alpha)$ is therefore a set of true $\in$-ordinals).

One can develop a theory of $E M$-sets and of their well-foundedness. If $\Sigma$ is well-founded (i.e. if $\mathcal{M}(\Sigma, \alpha)$ is well-founded for every ordinal $\alpha$ ) and if $\alpha$ is a limit ordinal, then $\mathcal{M}(\Sigma, \alpha)$ is isomorphic to a $\left\langle L_{\lambda}, \in\right\rangle$. Moreover, if $I(\Sigma, \kappa)$, with $\kappa>\omega$ an uncountable cardinal, is unbounded in the class of ordinals of $\mathcal{M}(\Sigma, \alpha)$ (and it is then the case for every ordinal $\alpha>\omega$ ), and if for every ordinal $\gamma<i_{\omega}$ (the $\omega$-th element of $I(\Sigma, \kappa)$ ) we have $\gamma \in \operatorname{Hull}^{\mathcal{M}}\left(\left\{i_{n}\right\}\right)$ (and it is then the case for every ordinal $\alpha>\omega)$, then $\mathcal{M}(\Sigma, \kappa)=\left\langle L_{\kappa}, \in\right\rangle, I(\Sigma, \kappa)$ is closed unbounded in $\kappa$ and if $\tau>\kappa>\omega$ then $I(\Sigma, \tau) \cap \kappa=I(\Sigma, \kappa)$.

Let us return to Silver indiscernibles. If such a $\Sigma$ exists, $S$ is defined by

$$
S=\bigcup\{I(\Sigma, \kappa): \kappa \text { uncountable cardinal }\} .
$$

The uniqueness of $S$ is a consequence of the unicity of such a $\Sigma$ :

Theorem. Such a $\Sigma$ is unique and is the set of $n$-ary sentences $\varphi$ s.t. $L_{\aleph_{\omega}} \models \varphi\left(\aleph_{1}, \ldots, \aleph_{n}\right)$. It is called "zero sharp" and written $0^{\#}$ (see below).

If there exists an uncountable limit cardinal $\kappa$ s.t. $\left\langle L_{\kappa}, \in\right\rangle$ possesses an uncountable set $I$ of indiscernibles, then $S$ exists. The existence of $S$ is also implied by large cardinal hypothesis as for instance:

Theorem. If there exists a $M C$ then $S$ exists and moreover $L_{\kappa} \prec$ $L_{\lambda}$ for every uncountable $\kappa<\lambda$.

The existence of $S$ under $M C$ means that after the first uncountable level $L_{\aleph_{1}}$ all the $L_{\kappa}$ share essentially the same first-order theory. $V$ 
transcends $L$, but in such a way that it makes $L$ as simple as possible, $L_{\aleph_{1}}=$ Hull $_{\aleph_{1}}\left(S \cap \aleph_{1}\right)$ determining the theory of $L$.

A deep consequence is that the truth in $L$ becomes definable in $V$. Indeed, let $\varphi\left(x_{1}, \ldots, x_{n}\right)$ be a formula. There exists an uncountable cardinal $\kappa$ s.t.

$$
\text { for all }\left(x_{i}\right) \in L_{\kappa}, L \models \varphi\left(x_{i}\right) \text { iff } L_{\kappa} \models \varphi\left(x_{i}\right) \text {. }
$$

As $L_{\kappa} \prec L_{\lambda}$ if $\kappa<\lambda$, we have

$$
L \models \varphi\left(x_{i}\right) \text { iff } L_{\lambda} \models \varphi\left(x_{i}\right) \text { for all } \lambda \geq \kappa .
$$

Now, we arithmetize the situation. Let $T=\left\{\ulcorner\varphi\urcorner: L_{\aleph_{1}} \models \varphi\right\}$ be the set of Gödel numbers of the $\varphi$ valid in $L_{\aleph_{1}}$ and therefore in all the $L_{\kappa}$ ( $\kappa$ uncountable) by elementarity. Then

$$
L \models \varphi \text { iff }\ulcorner\varphi\urcorner \in T
$$

defines the truth in $L$. This is not in contradiction with Gödel-Tarski uncompleteness theorems since $\aleph_{1}$ and $T$ are not definable in $L$ and therefore the truth of $L$ is not definable in $L$.

\subsection{The set $0^{\#}$}

As $L_{\aleph_{\omega}} \prec L$ and $\aleph_{i} \in S$ for $i>0$ in $\omega$, we can represent the indiscernibles in formulae by some of the $\aleph_{i}$ 's and restrict to $L_{\aleph_{\omega}}$, which contains all the $\aleph_{i}$ 's. Then, $L \models \varphi\left(x_{i}\right)$ for $x_{i} \in S$ iff $L_{\aleph_{\omega}} \models \varphi\left(\aleph_{i}\right)$. Solovay called $0^{\#}$ (zero sharp) the set (if it exists) defined by

$$
0^{\#}=\left\{\varphi: L_{\aleph_{\omega}} \models \varphi\left(\aleph_{i}\right)\right\}
$$

which is the set of formulae true on the indiscernibles of $L$. Via Gödelization $0^{\#}$ becomes a set of integers (also written $0^{\#}$ ) and can therefore be coded by a real (also written $0^{\#}$ ).

We must emphasize the fact that, as $L_{\aleph_{1}} \prec L$, every constructible set $x \in L$ which is definable in $L$ is countable since its definite description is valid in $L_{\aleph_{1}}$ by elementarity and therefore $x \in L_{\aleph_{1}}$. More generally for every infinite constructible set $x \in L$ we have $|\mathcal{P}(x)|^{L}=|x|$. Since the existence of a measurable cardinal implies that $0^{\#}$ exists, we have:

Corollary. If there exists a $M C$, the constructible continuum $\mathbb{R}^{L}$ is countable. 
Via arithmetization through Gödel numbers, the non constructible set $0^{\#}$ can be considered as a very special subset $\notin L$ of $\omega=\mathbb{N}$ or as a very special real number coding the truth in $L$. Its existence implies that every uncountable cardinal $\kappa$ of $V$ is an indiscernible of $L$ and shares all large cardinal axioms verified by $L$.

A property equivalent to the existence of $0^{\#}$ is the non rigidity of $L$ :

Theorem (Kunen). $0^{\#}$ exists iff there exists a non trivial elementary embedding $j: L \prec L$ (this presuppose $V \neq L$ and $j$ non trivial, see below).

Indeed, as $\operatorname{Hull}^{L}(S)=L$, for every $x \in L$ there exists a Skolem term $t$ s.t. $x=t\left(i_{\alpha_{1}}, \ldots, i_{\alpha_{n}}\right), i_{\alpha}$ being the $\alpha$-th element of $S . j$ is then simply defined by the shift on indiscernibles

$$
j(x)=j\left(t\left(i_{\alpha_{1}}, \ldots, i_{\alpha_{n}}\right)\right)=t\left(i_{\alpha_{1}+1}, \ldots, i_{\alpha_{n}+1}\right) .
$$

One shows that it is an elementary embedding and, as $j\left(i_{0}\right)=i_{1} \neq i_{0}$, $j$ is non trivial.

By the way, this proves again that $V \neq L$ since another theorem of Kunen proves that

$$
Z F C \vdash \text { there exists no } j: V \prec V \text {. }
$$

The existence of $0^{\#}$ is a principle of transcendence of $V$ over $L$ expressing that $V$ is very different from $L$. If $0^{\#}$ doesn't exist, then $V$ looks like $L$ ( $L$ is a good approximation of $V$ ) according to the result:

Covering lemma (Jansen). If $0^{\#}$ doesn't exist, then if $x$ is an uncountable set of ordinals there exists a constructible set $y \supseteq x$ of the same cardinality as $x$. So, every set $x$ of ordinals can be covered by a constructible set $y \supseteq x$ of cardinality $|y|=|x| \aleph_{1}$.

Corollary. If $0^{\#}$ doesn't exist, the covering lemma implies that, for every limit singular cardinal $\kappa$ of $V$, we have $\left(\kappa^{+}\right)^{L}=\kappa^{+}$, which shows that $V$ and $L$ are quite similar.

Indeed (see Jech [1978], p. 358), if $\lambda=\left(\kappa^{+}\right)^{L}$ and if $\lambda<\kappa^{+}$, then $|\lambda|=\kappa$. But as $\kappa$ is singular, we would have $\operatorname{cf}(\lambda)<|\lambda|$ and this is impossible since $\lambda$ is regular in $L$ and $\lambda \geq \omega_{2}$. For, if $x$ is an unbounded subset of $\lambda$ of cardinal $|x|=\operatorname{cf}(\lambda)$, it can be covered by a constructible subset $y \in L$ of $\lambda$ of cardinal $|y|=|x| \aleph_{1}$ and, as $\lambda$ is regular in $L,|y|=|\lambda|$. So, $|\lambda|=\aleph_{1}$. cf $(\lambda)$ and, as $\lambda \geq \omega_{2}$, $|\lambda|=\operatorname{cf}(\lambda)$. 
Corollary. If $G C H$ fails at a strong limit singular cardinal, then $0^{\#}$ exists.

Indeed, if $0^{\#}$ doesn't exist, for such a singular cardinal $\kappa$ we have $\left(\kappa^{+}\right)^{L}=\kappa^{+}$. As $L$ satisfies $G C H,\left(2^{\kappa}\right)^{L}=\left(\kappa^{+}\right)^{L}=\kappa^{+} 22$ Now, $\kappa$ is a strong limit by hypothesis (i.e. $\lambda<\kappa \Rightarrow 2^{\lambda}<\kappa$ ) and this implies $\kappa^{\operatorname{cf}(\kappa)}=2^{\kappa}$ and moreover, since $\kappa$ is singular and therefore $\operatorname{cf}(\kappa)<|\kappa|, 2^{\operatorname{cf}(\kappa)}<\kappa$. Let $x \in A=[\kappa]^{\operatorname{cf}(\kappa)}$ be a subset of $\kappa$ of cardinal $\mathrm{cf}(\kappa)$. It is covered by a constructible subset $y \in L$ of $\kappa$ of cardinal $|y|=\lambda=\aleph_{1}$. $\operatorname{cf}(\kappa)$. Then $A$ can be covered by the union of the $[y]^{\operatorname{cf}(\kappa)}$ for such $Y$. But $\left|[y]^{\operatorname{cf}(\kappa)}\right|=\lambda^{\operatorname{cf}(\kappa)}=\left(\aleph_{1} \cdot \operatorname{cf}(\kappa)\right)^{\operatorname{cf}(\kappa)}=2^{\operatorname{cf}(\kappa)}$, and by hypothesis $2^{\mathrm{cf}(\kappa)}<\kappa$. Now, there exist at most $|\mathcal{D}(\kappa)|$ such $y 23$ and $|\mathcal{D}(\kappa)|=\left(\kappa^{+}\right)^{L}=\kappa^{+}$. All this implies $|A|=\kappa^{\mathrm{cf}(\kappa)}=\kappa^{+}$. As $\kappa^{\operatorname{cf}(\kappa)}=2^{\kappa}$, we have $2^{\kappa}=\kappa^{+}$, that is $G C H$.

There are many ways for insuring that $0^{\#}$ exists, for instance $P F A$ since PFA implies the failure of $\square_{\kappa}$ for every $\kappa$ (Todorcevic, 1980). More generally, many results show that a failure of $\square_{\kappa}$ is linked with $L C A s$. For instance:

- Jensen: if $\square_{\kappa}$ fails for some singular $\kappa$, there exists an inner model $M$ with a strong cardinal.

- Solovay: if $\kappa$ is supercompact, then $\square_{\lambda}$ fails for every $\lambda>\kappa$.

This is related to the fact that properties such as the covering between $V$ and $M$ allow to reflect $\square_{\kappa}$ from $M$ to $V$ and therefore, if $\square_{\kappa}$ fails in $V$ for $\kappa$ singular it is because there exist some $L C s$ violating the covering lemma.

When $0^{\#}$ exists, a very interesting structure to look at is $L\left[0^{\#}\right]$. It can be shown 24 that $L\left[0^{\#}\right]$ has a fine structure, satisfies the Global Square property $\forall \kappa \square_{\kappa}$ and, for every singular cardinal $\kappa$ of $V,\left(\kappa^{+}\right)^{L\left[0^{\#}\right]}=\kappa^{+}$. Iterating the sharp operation, one can get an increasing sequence of models $M$ mildly transcendent over $L$ which have also a fine structure, satisfy the Global Square property and, for every singular cardinal $\kappa$ of $V,\left(\kappa^{+}\right)^{M}=\kappa^{+}$, and are smaller than the first inner model possessing a measurable cardinal. Under the hypothesis that $a^{\#}$ exists for every set of ordinals $a\left(a^{\#}\right.$ is defined as $0^{\#}$ but

\footnotetext{
${ }^{22} \mathrm{As}$, if $0^{\#}$ exists, $\left(2^{\kappa}\right)^{L}=\kappa$ for every infinite cardinal since $\kappa^{+}$is inaccessible in $L$, $\left(2^{\kappa}\right)^{L}=\kappa^{+}>\kappa$ is a counter exemple when $0^{\#}$ doesn't exist.

${ }^{23}$ Recall that $\mathcal{D}(x)$ is the set of constructible parts of $x$ (see above).

${ }^{24}$ See e.g. Steel [2001].
} 
in $L[a]$ ), one can even go beyond this limit, up to the existence of a supercompact cardinal.

\section{3 $\quad 0^{\#}$ and the hierarchical structure of $V$ be- yond $L$.}

The equivalence between the existence of $0^{\#}$ and the existence of a non trivial elementary embedding $j: L \prec L$ enables to clarify the structure of $V$ beyond $L 25$. Let $\kappa=\operatorname{crit}(j)$ and let $\mathcal{U}$ be the set of subsets $u \subseteq \kappa$ s.t. $u \in L$ (i.e. $u \in L \cap \mathcal{P}(\kappa))$ and $\kappa \in j(u)$. $\mathcal{U}$ is trivially a filter. It is an ultrafilter since for every $u \in L \cap \mathcal{P}(\kappa)$ either $\kappa \in j(u)$ or $\kappa \notin j(u)$. It is a free ultrafilter since if $u \subset \kappa$ is bounded, then $\kappa \notin u, j(u)=u$, $\kappa \notin j(u)$ and therefore $u \notin \mathcal{U}$. Moreover, it is $\kappa$-complete w.r.t. $L$ since if $u_{\alpha} \in \mathcal{U} \cap L$ is a family with $\alpha<\beta<\kappa$ then $\bigcap_{\alpha<\beta} u_{\alpha} \in \mathcal{U}$. One can show that the ultrapower $L^{\mathcal{U}}$ is well-founded. Due to Łoš theorem, the embedding $i: L \prec L^{\mathcal{U}}$ is elementary. But the Mostowski collapsing lemma implies that $\left\langle L^{\mathcal{U}}, \in_{\mathcal{U}}\right\rangle \simeq\left\langle M_{\mathcal{U}}, \in\right\rangle$ for some transitive inner model $M_{\mathcal{U}}$. But necessarily $M_{\mathcal{U}}=L$ by minimality of $L$ and via this isomorphism $i: L \prec L^{\mathcal{U}}$ becomes an elementary embedding $j: L \prec L$. It can be shown that, if $\lambda=\left(\kappa^{+}\right)^{L}$ then $\mathcal{M}=\left\langle L_{\lambda}, \in, \mathcal{U}\right\rangle$ is a model of $Z F-\{$ Power Set axiom $\}$ where $\kappa$ becomes the largest cardinal, $\mathcal{U}$ remains a free ultrafilter $\kappa$-complete with "good" technical properties ("normality" and "amenability"). Such a procedure can be iterated on the ordinals. Starting from a $\mathcal{M}_{0}=\left\langle L_{\lambda_{0}}, \in, \mathcal{U}_{0}\right\rangle$, one gets a $\mathcal{M}_{1}=\left\langle L_{\lambda_{1}}, \in, \mathcal{U}_{1}\right\rangle$, etc. The successive $\mathcal{M}_{\alpha}$ yield a sequence of critical cardinals $\kappa_{\alpha}$ which are indiscernibles for $L$.

\section{Determination and reflection phe- nomena}

To measure the size of large cardinals, the best way is to use associated reflection phenomena which are of a very deep philosophcal value 26 Intuitively, reflection means that the properties of the whole universe $V$ are reflected in sub-universes. As was emphasized by Matthew Foreman (1998, p. 6):

\footnotetext{
${ }^{25}$ See e.g. Schimmerling [2001].

${ }^{26}$ See Martin-Steel [1989], Patrick Dehornoy [1989].
} 
"Any property that holds in the mathematical universe should hold of many set-approximations of the mathematical universe."

Definition. A cardinal $\chi$ reflects a relation $\Phi(x, y)$ defined on ordinals if every solution $y \geq \chi$ parametrized by $x<\chi$ can be substituted for by a solution $y<\chi$ :

$$
\forall \alpha(\in O n)<\chi\left[\exists \beta \geq \chi \Phi(\alpha, \beta) \Rightarrow \exists \beta^{*}<\chi \Phi\left(\alpha, \beta^{*}\right)\right] .
$$

Let $j$ be an elementary embedding $j: M \prec M^{*}$. $\chi=\operatorname{crit}(j)$ is a large - in fact at least measurable - cardinal, which increases indefinitely when $M^{*}$ moves near to $M$, the limit $M^{*}=M$ being inconsistent according to Kunen theorem.

To see that it is a reflection phenomenon, let $\Phi(\alpha, \chi)$ be a relation that holds in $M$ for $\alpha<\chi$. If $M^{*}$ is sufficiently close to $M$ for $\Phi(\alpha, \chi)$ to remain true in $M^{*}$, then $M^{*} \models \exists(x<j(\chi)) \Phi(\alpha, x)$ (it is sufficient to take $x=\chi$ ). But, according to the elementarity of the embedding $j$, this is equivalent to $M \models \exists(x<\chi) \Phi(\alpha, x)$.

To go beyond measurable cardinals, specialists use the following technique. Let $V_{\alpha}$ be the cumulative hierarchy of sets up to level $\alpha$. For $\chi$ critical (and therefore measurable), one has $V_{\chi}^{M^{*}}=V_{\chi}^{M}$ (that is the equality of $M$ and $M^{*}$ up to level $\chi$ ).

Definition. The cardinal $\chi$ is called superstrong in $M$ if there exists an elementary embedding $j$ s.t. $V_{j(\chi)}^{M^{*}}=V_{j(\chi)}^{M}$ (that is $V_{j(\chi)}^{M^{*}} \subset M$ and $M=M^{*}$ up to $j(\chi)$ and not only up to $\left.\chi\right)$.

Between measurable and superstrong cardinals, Hugh Woodin introduced another class of large cardinals.

Definition. A cardinal $\delta$ is called a Woodin cardinal if for every map $F: \delta \rightarrow \delta$, there exists $\kappa<\delta$ and an elementary embedding $j$ of critical ordinal $\kappa$ s.t. $\left.F\right|_{\kappa}: \kappa \rightarrow \kappa$ and $V_{j(F(\kappa))}^{M^{*}}=V_{j(F(\kappa))}^{M}$ (that is $M=M^{*}$ up to $\left.j(F(\kappa))\right)$.

Woodin has shown that:

1. if $\delta$ is a Woodin cardinal, there exist infinitely many smaller measurable cardinals $\chi<\delta$,

2. if $\lambda$ is a superstrong cardinal, there exist infinetely many smaller Woodin cardinals $\delta<\lambda$.

A key result is the Martin-Steel theorem which evaluates exactly the "cost" of determinacy: 
Martin-Steel theorem (1985). If there exist $n$ Woodin cardinals $\delta_{i}, i=1, \ldots, n$, dominated by a measurable cardinal $\kappa\left(\kappa>\delta_{i}\right.$ for all $i$ ), then $Z F C \vdash \operatorname{Det}\left(\Pi_{n+1}^{1}\right)$.

The converse is due to Woodin.

Corollary. If there exists a countable infinity of Woodin cardinals dominated by a measurable cardinal, in particular if there exists a superstrong cardinal $\lambda$, then Projective Determinacy is valid (all the projective subsets of $\mathbb{R}$ are determined).

Projective Determinacy is also valid under PFA (Woodin, see above).

It is for this reason that specialists consider that $Z F C+$ Projective Determinacy is a "good" axiomatic for $\mathbb{R}$. We must also emphasize the:

Martin-Steel-Woodin theorem (1987). If there exists a countable infinity of Woodin cardinals dominated by a measurable cardinal, in particular if there exists a superstrong cardinal $\lambda$, then $L(\mathbb{R})$ (the smallest inner model of $V$ containing the ordinals $O n$ and $\mathbb{R}$, see above) satisfies the axiom of complete determinacy $A D$ : every $A \subseteq \mathbb{R}$ is determined. (This result is stronger than the previous one since $\mathcal{P}(\mathbb{R}) \cap L(\mathbb{R})$ is a larger class than the projective class.)

$A D$ is incompatible with $A C$ since $A C$ enables the construction of a non determined well ordering on $\mathbb{R}$ (see above) 27

But the most significative results concern perhaps the situation where no property of $\mathbb{R}$ could be further modified in a forcing extension. In that case, the theory of the continuum becomes "rigid". Woodin and Shelah have shown that it is possible to approximate this ideal goal if there exists a supercompact cardinal $\kappa . \kappa$ is $\gamma$ supercompact if there exists an elementary embedding $j: V \prec M$ s.t. $\operatorname{crit}(j)=\kappa, \gamma<j(\kappa)$ and $M^{\gamma} \subseteq M . \kappa$ is supercompact if it is $\gamma$-supercompact for every $\gamma \geq \kappa(\kappa$ is $\kappa$-supercompact iff it is measurable) 28 Such a deep result clarifies the nature of the axioms which are needed for a "good" theory of the continuum.

\section{Woodin's $\Omega$-logic}

Large cardinal axioms ( $L C A s)$ can decide some properties of regularity of $\mathbb{R}$, but they cannot settle $C H$ since a "small" forcing (adding

\footnotetext{
${ }^{27}$ So, the inner model $L(\mathbb{R})$ of a $Z F C$-model $V$ can violate $A C$.

${ }^{28}$ See Dehornoy [2003].
} 
$\aleph_{2}$ new subsets to $\omega$ ) is sufficient to force $\neg C H$ from a $C H$-model and such a small forcing remains possible irrespective of what $L C A s$ are introduced (Levy-Solovay theorem). We need therefore a new strategy. As we have just seen, the most natural one is to try to make the properties of the continuum immune relatively to forcing, that is to make the continuum in some sense "rigid". The deepest contemporary results in this perspective are provided by Woodin's extraordinary recent works on $\Omega$-logic and the negation of $\mathrm{CH}$.

We look for theories sharing some absoluteness properties relatively to forcing. This is called "conditional generic absoluteness" 29

The fragment of $V$ where $C H$ "lives" naturally is $\left(H_{2}, \in\right)$ where $\left(H_{k}, \in\right)$ is the set of sets $x$ which are hereditary of cardinal $|x|<\aleph_{k}$. The fragment $\left(H_{0}, \in\right)=V_{\omega}$ is the set of hereditary finite sets and, with the axioms $Z F$ minus the axiom of infinity, is equivalent to first order arithmetic $\langle\omega=\mathbb{N},+, ., \in\rangle$ with Peano axioms. In one direction, $\mathbb{N}$ can be retrieved from $H_{0}$ using von Neumann's construction of ordinals and, conversely, $H_{0}$ can be retrieved from $\mathbb{N}$ via Ackermann's trick: if $p, q$ are integers, $p \in q$ iff the $p$-th digit in the binary extension of $q$ is 1. For first order arithmetic, Peano axioms are "empirically" and practically complete in spite of Gödel incompleteness theorem. The following classical result expresses their "rigidity":

Schönfield theorem. $H_{0}$ is absolute and a fortiori forcing-invariant. Incompleteness cannot be manifested in it using forcing.

We can therefore consider $Z F C$ as a "good" theory for first order arithmetic. But it is no longer the case for larger fragments of $V$.

The fragment $\left(H_{1}, \in\right)$ of $V$ composed of countable sets of finite ordinals is isomorphic to $\langle\mathcal{P}(\omega)=\mathbb{R}, \omega,+, ., \in\rangle$ and corresponds to second order arithmetic (i.e. analysis). The definable subsets $A \subseteq$ $\mathcal{P}(\omega)$ are the projective subsets 30 and therefore $H_{1}$ can be considered as the fragment of $V$ where the projective sets live. We have seen that to settle and "freeze" most of its higher order properties (regularity of projective sets) w.r.t. forcing, we need $L C A s$ and in particular PD.

As is emphasized by Woodin (2003, quoted in Dehornoy [2003]):

"Projective Determinacy settles (in the context of $Z F C$ ) the classical questions concerning the projective sets and

\footnotetext{
${ }^{29}$ See Steel [2004]: "Generic absoluteness and the continuum problem".

${ }^{30} A \subseteq \mathcal{P}(\omega)$ is definable in $H_{1}$ (with parameters in $H_{1}$ ) iff there exists a first-order formula $\varphi(x, y)$ and a parameter $b \in \mathcal{P}(\omega)$ s.t. $A=\left\{a \in \mathcal{P}(\omega) \mid H_{1} \models \varphi(a, b)\right\}$. If $\pi$ : $\mathcal{P}(\omega) \rightarrow[0,1] \simeq \mathbb{R}$ is given by $\pi(a)=\sum_{i \in a} \frac{1}{2^{i}}$, then $X \subseteq[0,1]$ is projective iff $A=\pi^{-1}(X)$ is definable in $H_{1}$.
} 
moreover Cohen's method of forcing cannot be used to establish that questions of second order number theory are formally unsolvable from this axiom. (...) I believe the axiom of Projective Determinacy is as true as the axioms of Number Theory. So I suppose that I advocate a position that might best be described as Conditional Platonism."

We have also seen that under the $L C A$ "there exists a proper class of Woodin cardinals" ( $P C W$ : for every cardinal $\kappa$ there exists a Woodin cardinal $>\kappa)$ we have:

Theorem (Woodin, 1984). $Z F C+P C W \vdash H_{1}$ is immune relatively to forcing in the sense its properties are forcing-invariant.

As $P C W$ implies at the same time $P D$ and forcing-invariance for $H_{1}$, it can be considered as a "good" theory, "empirically" and "practically" complete (marginalizing incompleteness) for $\left(H_{1}, \in\right)$, that is for analysis (second order arithmetic). $P C W$ implies the generic completeness result that all the $L[\mathbb{R}]$ of generic extensions $V[G]$ are elementary equivalent.

The idea is then to try to generalize these types of absoluteness properties relative to forcing. The general strategy for deciding that way $Z F C$-undecidable properties $\varphi$ in a fragment $H$ of $V$ is described in the following way by Patrick Dehornoy (2003):

"every axiomatization freezing the properties of $H$ relatively to forcing (i.e. neutralizing forcing at the level $H$ ) implies $\varphi$ ".

The main problem tackled by Woodin was to apply this strategy to the fragment $\left(H_{2}, \in\right)$ of $V$ which is associated to the set $\mathcal{P}\left(\omega_{1}\right)$ of countable ordinals. $\mathcal{P}\left(\omega_{1}\right)$ is not $\mathcal{P}(\mathbb{R})$ if $\neg C H$ is satisfied, but nevertheless it is possible to code $C H$ by an $H_{2}$-formula $\varphi_{C H}$ s.t. $H_{2} \models \varphi_{C H}$ is equivalent to $C H 31$ The problem with $H_{2}$ is that "small" forcings preserve LCAs 32 and in particular (Levy-Solovay theorem, 1967) a small forcing of cardinal $\aleph_{2}$ that enables to violate $\mathrm{CH}$ by adding $\aleph_{2}$ subsets to $\mathbb{N}$ preserves $L C A s$. Therefore $\mathrm{H}_{2}$ cannot

\footnotetext{
${ }^{31}$ The point is rather technical. Woodin has shown that if $\neg C H$ is valid (i.e. $\mathbb{R}>\omega_{1}$ ), $\mathcal{P}(\mathbb{R}) \notin H_{2}$ and is already too big for freezing (neutralizing the effects of forcing) the fragments of $V$ containing it.

${ }^{32}$ Large cardinal axioms are axioms of the form $A=\exists \kappa \psi(\kappa)$ which share the property that if $V \vDash A$ then the cardinal $\kappa$ is inaccessible and $\psi(\kappa)$ is forcing-invariant for every forcing extension $V[G]$ of forcing cardinal $<\kappa$ ("small" forcings).
} 
be rigidified by $L C A s 33$ Whatever the large cardinal hypothesis $A$ may be, there will be always generic extensions $M$ and $N$ of $V$ both satisfying $A$ such that $M \models C H$ and $N \models \neg C H$. As $C H$ is equivalent to a $\Sigma_{2}^{1}$ formula, $M$ and $N$ cannot be elementary equivalent from the $\Sigma_{2}^{1}$ level.

Woodin's fundamental idea to overcome the dramatic difficulties of the problem at the $\mathrm{H}_{2}$ level was to strengthen logic by restricting the admissible models and constructing a new logic adapted to forcinginvariance or "generic invariance". As he explains in his key paper on "The continuum hypothesis" (2001, p. 682):

"As a consequence (of generic invariance), any axioms we find will yield theories for $\left\langle H\left[\omega_{2}\right], \in\right\rangle$, whose 'completeness' is immune to attack by applications of Cohen's method of forcing, just as it is the case for number theory."

In a first step, he introduced the notion of $\Omega$-validity $\models_{\Omega}$ also called in a first time $\Omega^{*}$-derivability $\vdash_{\Omega^{*}}$.

Definition. $T$ being a theory in $Z F C$, we have $T \models_{\Omega} \varphi$ iff $\varphi$ is valid in every generic extension where $T$ is valid, that is iff for every generic extension $V[G]$ and every level $\alpha,\left(V_{\alpha}\right)^{V[G]} \models T$ implies $\left(V_{\alpha}\right)^{V[G]} \models \varphi$.

Of course $\models$ implies $\models_{\Omega}$. But the converse is trivially false: there exists $\Omega$-valid formulae which are undecidable in $Z F C$, for instance Con $(Z F C)$. Indeed, if $\left(V_{\alpha}\right)^{V[G]} \models Z F C$ then $\left(V_{\alpha}\right)^{V[G]}$ is a model of $Z F C$ and $\left(V_{\alpha}\right)^{V[G]} \models \operatorname{Con}(Z F C)$ So, $Z F C \models_{\Omega} \operatorname{Con}(Z F C)$, but of course (Gödel) $Z F C \not \models \operatorname{Con}(Z F C)$.

It must be emphasized that $\Omega$-validity doesn't satisfy the compacity property: there exist theories $T$ and formulae $\varphi$ s.t. we have $T \models_{\Omega} \varphi$ even if for every finite subset $S \subset T$ we have $S \nvdash_{\Omega} \varphi 34$

By construction, $\Omega$-validity $\models_{\Omega}$ is itself forcing-invariant 35

Theorem $(Z F C+P C W)$. If $V \models " T \models_{\Omega} \varphi$ " then $V[G] \models$ " $T \models_{\Omega} \varphi$ " for every generic extension of $V$.

Woodin investigated deeply this new "strong logic". In particular he was able to show that, under suitable $L C A s, C H$ "rigidifies" $V$ at the $\Sigma_{1}^{2}$-level $\left(\Sigma_{1}\right.$ formulae for $\left.V_{\omega+2}\right)$ :

Theorem (Woodin, 1984). Under $P C W_{\text {meas }}$ (there exists a proper class of measurable Woodin cardinals) and $\mathrm{CH}, \Omega$-logic is

\footnotetext{
${ }^{33}$ See Dehornoy (2003).

${ }^{34}$ See Bagaria et al. [2005].

${ }^{35}$ See Woodin [2004].
} 
generically complete at the $\Sigma_{1}^{2}$-level: for every $\varphi$ of complexity $\Sigma_{1}^{2}$ either $Z F C+C H \models_{\Omega} \varphi$ or $Z F C+C H \models_{\Omega} \neg \varphi$. All generic extensions $M$ and $N$ of $V$ satisfying both $C H$ are $\Sigma_{1}^{2}$ elementary equivalent.

The metamathematical meaning of this result of conditional generic absoluteness is that if a problem is expressed by a $\Sigma_{1}^{2}$-formula $\varphi$ then it is "settled by $C H$ " and immunized against forcing under appropriate LCAs. But:

Theorem (Abraham, Shelah). This is false at the $\Sigma_{2}^{2}$ level. For every large cardinal hypothesis $A$ there exist generic extensions $M$ and $N$ satisfying both $C H$ s.t. in $M$ there exist a $\Sigma_{2}^{2}$-wellorder of $\mathbb{R}$ while in $N$ all the $\Sigma_{2}^{2}$-subsets of $\mathbb{R}$ are Lebesgue measurable.

In a second step, Woodin interpreted the $\Omega$-validity $T \models_{\Omega} \varphi$ as the semantic validity for an $\Omega$-logic whose syntactic derivation $T \vdash_{\Omega} \varphi$ had to be defined. His idea was to witness the $\Omega$-proofs by particular sets that, under $P C W$, generalize the projective sets and can be interpreted without ambiguity in every generic extension. It is the most difficult part of his work, not only at the technical level but also at the philosophical level. The definition (under $P C W$ ) is the following:

Definition $(P C W) . T \vdash_{\Omega} \varphi$ iff there exists a universally Baire $(U B)$ set $A \subseteq \mathbb{R}$ s.t. for every $A$-closed countable transitive model (ctm) $M$ of $T$ we have $M \models \varphi$ (in other words $M \models$ " $T \models_{\Omega} \varphi$ ").

$A \subseteq \mathbb{R}$ is $U B$ if for every continuous map $f: K \rightarrow \mathbb{R}$ with source $K$ compact Hausdorff, $f^{-1}(A)$ has the Baire property (there exists an open set $U$ s.t. the symetric difference $f^{-1}(A) \Delta U$ is meager). If $A \subseteq \mathbb{R}$ is $U B$ it is interpreted canonically in every generic extension $V[G]$ as $A_{G} \subseteq \mathbb{R}^{V[G]}$. This is due to the fact that there exists a tree presentation of $A$. One identifies $\mathbb{R}$ with $\omega^{\omega}$ and one considers trees $T \subset(\omega \times \gamma)^{\omega}$ and the projections $p[T]$ on $\omega^{\omega}$ of their infinite branches:

$$
p[T]=\left\{x \in \omega^{\omega} \mid \exists z \in \gamma^{\omega} \text { with }\left(\left.x\right|_{n},\left.z\right|_{n}\right) \in T, \forall n \in \omega\right\} .
$$

$A \subseteq \mathbb{R}$ is $U B$ iff there exist trees $T$ and $S$ s.t. $p[T]=A$ and $p[S]=\omega^{\omega}-A$ in every generic extension $V[G] . p[T]$ yields a canonical interpretation of $A$ in every generic extension $V[G]$. A ctm $M$ is called $A$-closed if, for every ctm $N \supseteq M, A \cap N \in N$, in particular for every generic extension $V[G]$ and $N=M[G]$ we have $A \cap M[G] \in M[G]$. If $A$ is Borelian, every ctm is always $A$-closed 36 But it is no longer the case for general $U B$ sets.

\footnotetext{
${ }^{36}$ If $M$ is $A$-closed for every $A$ of $\Pi_{1}^{1}$-complexity, then $M$ is well-founded.
} 
As far as, in the definition of $T \vdash_{\Omega} \varphi$, the class of admissible models is restricted to $A$-closed ctms, logic becomes strengthened. Of course, $T \vdash \varphi$ implies $T \vdash_{\Omega} \varphi$, but the converse is false for the same reasons as for $\vDash_{\Omega}$. Indeed, $Z F C \vdash_{\Omega} \operatorname{Con}(Z F C)$ because every suitable ctm provides a model of $Z F C$ and validates therefore Con $(Z F C)$.

More technically, what is really needed for the definition $T \vdash_{\Omega} \varphi$ are $U B$ sets $A \subseteq \mathbb{R}$ sharing the following two properties:

1. $L(A, \mathbb{R}) \vDash A D^{+}$, where $A D^{+}$is a strengthening of the axiom of determinacy saying that not only all $A \subseteq \mathbb{R} \simeq \omega^{\omega}$ are determined, but also all the $\pi^{-1}(A)$ for all maps $\pi: \lambda^{\omega} \rightarrow \omega^{\omega}$ with an ordinal $\lambda<\mathfrak{c}^{+}$

2. every $A \subset \mathcal{P}(\mathbb{R}) \cap L(A, \mathbb{R})$ is $U B 37$

$P C W$ implies these two properties and is therefore a good hypothesis.

It must be emphasized that this definition of $\Omega$-provability is very original. As explain Joan Bagaria, Neus Castells and Paul Larson in their " $\Omega$-logic primer":

"The notion of $\Omega$-provability differs from the usual notions of provability, e.g., in first-order logic, in that there is no deductive calculus involved. In $\Omega$-logic, the same $U B$ set may witness the $\Omega$-provability of different sentences. For instance, all tautologies have the same proof in $\Omega$-logic, namely $\emptyset$. In spite of this, it is possible to define a notion of height of proof in $\Omega$-logic."

As Patrick Dehornoy explained to me (private communication), in $\Omega$ logic a proof $\vdash_{\Omega} \varphi$ is a certificate of some property of the formula $\varphi$. This witnessing is no longer a derivation iterating syntactic rules but a $U B$ subset of $\mathbb{R}$. What is common to classical and $\Omega$-logics is that a very "small" object endowed with a precise internal structure warrants the validity of $\varphi$ in a lot of immensely large models.

Woodin proved that $\Omega$-logic is sound: if $T \vdash_{\Omega} \varphi$ then $T \models_{\Omega} \varphi$, i.e. (under $P C W)$ if $\vdash_{\Omega} \varphi$ then $\models \varphi$ in all $Z F C$-models $\left(V_{\alpha}\right)^{V[G]}$. He then formulated the main conjecture:

$\Omega$-conjecture (1999). $\Omega$-logic is complete: if $\models_{\Omega} \varphi$ then $\vdash_{\Omega} \varphi$. $\square$ As he emphasized in Woodin (2002, p. 517):

\footnotetext{
${ }^{37}$ See Woodin [2000].
} 
"If the $\Omega$-conjecture is true, then generic absoluteness is equivalent to absoluteness in $\Omega$-logic and this in turn has significant metamathematical implications".

Indeed (Dehornoy, 2007), the $\Omega$-conjecture means that any formula $\varphi$ valid in a lot of immensely large models satisfying $L C A s$ are certified by $U B$ subsets of $\mathbb{R}$. The key fact proved by Woodin is the link of the concept of $\Omega$-derivability with the existence of canonical models for $L C A s$ (that is models which are in a certain way minimal and universal, as $L$ for $Z F C+C H$ ). The $\Omega$-conjecture expresses essentially the hypothesis that every $L C A$ admits a canonical model.

Theorem. $\vdash_{\Omega} \varphi$ iff $Z F C+A \vdash \varphi$ for every large cardinal axiom $A$ admitting a canonical model.

Now, the key point is that when $\mathrm{H}_{2}$ is rigidified, $\mathrm{CH}$ becomes automatically false.

"If the theory of the structure $\left\langle\mathcal{P}\left(\omega_{1}\right), \omega_{1},+, ., \in\right\rangle$ is to be resolved on the basis of a good axiom then necessarily $\mathrm{CH}$ is false."

The idea is that if the theory $T$ of $\mathcal{P}\left(\omega_{1}\right)$ is completely unambiguous in the sense that there exists an axiom $A$ s.t. $T \models \varphi$ iff $A \models_{\Omega}$ " $T \models \varphi$ ", then $C H$ is necessarily false since the theory of $\mathcal{P}(\mathbb{R})$ cannot share this property.

Woodin theorem (2000, under $P C W$ ). (i) For every "solution" for $\mathrm{H}_{2}$ (that is axioms freezing the properties of $\mathrm{H}_{2}$ w.r.t. forcing) based on an $\Omega$-complete axiom $A$ (i.e. for every $\varphi \in H_{2}$, either $Z F C+$ $A \vdash_{\Omega}$ " $\left(H_{2}, \in\right) \models \varphi$ " or $Z F C+A \vdash_{\Omega}$ " $\left(H_{2}, \in\right) \models \neg \varphi$ "), $C H$ is false. (ii) If the $\Omega$-conjecture is valid, every "solution" for $\mathrm{H}_{2}$ is based on an $\Omega$-complete axiom and therefore $\mathrm{CH}$ is false.

The proof uses Tarski results on the impossibility of defining truth and is quite interesting (Woodin 2001, p. 688). Let

$$
\Gamma=\left\{\ulcorner\varphi\urcorner: Z F C+A \vdash_{\Omega} \text { " }\left(H_{2}, \in\right) \models \varphi "\right\}
$$

be the (extremely complicated) set of Gödel numbers of the sentences $\Omega$-valid in $H_{2}$. By hypothesis, $\Gamma$ is $\Omega$-recursive in the sense there exists a $U B$ set $B$ s.t. $\Gamma$ is definable and recursive in $L(B, \mathbb{R})$. Now, $P C W$ implies that $\Gamma$ being $\Omega$-recursive, it is definable in $\left(H\left(\mathfrak{c}^{+}\right), \in\right)$. If $C H$ would be valid, then $\mathfrak{c}=\omega_{1}, H\left(\mathfrak{c}^{+}\right)=H_{2}$ and $\Gamma$ would be definable in $\mathrm{H}_{2}$, which would violate Tarski theorem.

It is in that sense Woodin (2001, p. 690) can claim: 
"Thus, I now believe the Continuum Hypothesis is solvable, which is a fundamental change in my view of set theory".

\section{Conclusion}

Hugh Woodin has already proved a great part of the $\Omega$-conjecture.

Other approaches to the continuum problem in the set theoretical framework of $L C A s$ have been proposed. One of the most interesting alternative is provided by Matthew Foreman's (2003) concept of generic large cardinal $(G L C)$ defined by elementary embeddings $j: V \prec M$ of $V$ in inner models $M$ not of $V$ itself but of generic extensions $V[G]$ of $V$. Such generic $L C A s$ can support rather $C H$ than $\neg C H$.

But all these results show what are the difficulties met in elaborating a "good" set theoretical determination of the continuum. The old Kantian opposition between "conceptual" (symbolic) and "intuitive", or, in Feferman's terms, between "determined" and "inherently vague", remains insuperable. They justify some sort of Gödel's platonism comprising additional axioms as some kind of "physical hypotheses". The nominalist antiplatonist philosophy of mathematics criticizing such axioms (in particular $L C A s$ ) as ontological naive beliefs must be reconsidered and substituted for a "conditional" platonism in Woodin's sense, a platonism which would be "conditional" to axioms which "rigidify" the continuum and make its properties forcinginvariant.

In my 1991, 1992 and 1995 papers on the continuum problem, I introduced the concept of "transcendental platonism". Classical platonism is a naïve realist thesis on the ontological independence of mathematical idealities, and as such is always dialectically opposed to antiplatonist nominalism. Even to day, the debates concerning the status of mathematical idealities remain trapped into the realist/nominalist dialectic 38 The main achievement of transcendentalism has been to overcome this scholastic antinomy between realism and nominalism and to show that mathematical and physical objectivity were neither ontological nor subjective. Objectivity is always transcendentally constituted and therefore conditional, relative to eidetico-constitutive rules. A platonism defined in terms of objectivity and not ontology, is a transcendental platonism immune to the classical aporias of metaphys-

\footnotetext{
${ }^{38}$ See e.g. Maddy [2005] on "naïve realism", "robust realism", "thin realism", etc.
} 
ical transcendent platonism. As far as the question of the continuum is concerned, the eidetico-constitutive rules are the axioms of set theory and transcendental platonism means that the continuum problem can have a well determined solution in a "rigid" universe where $\mathbb{R}$ become conditionally generically absolute. I think that Woodin's conditional platonism can therefore be considered as a transcendental platonism relative to the continuum problem.

\section{References}

[1] Bagaria, J., Castells, N., Larson, P., 2005. "An $\Omega$-logic Primer", Set Theory, CRM 2003-2004, Birkhauser (2006), 1-28.

[2] Becker, H., 1992. "Descriptive Set Theoretic Phenomena in Analysis and Topology", STC [1992], 1-25.

[3] Bell, J., 2005. The Continuous and the Infinitesimal in Mathematics and Philosophy, Polimetrica, http://www.polimetrica.com/categories/02cat.html.

[4] Bellotti, L., 2005. "Woodin on the Continuum Problem: an overview and some objections", Logic and Philosophy of Science, III, 1.

[5] Benacerraf, P., Putnam, H. (eds.), 1964. Philosophy of Mathematics: Selected Readings, Prentice Hall, Englewood Ciffs, NewJersey.

[6] Breysse, O., De Glas, M., 2007. "A New Approach to the Concepts of Boundary and Contact: Towards an Alternative to Mereotopology", Fundamenta Informaticae, 78, 2, 217-238.

[7] Chihara, Ch., S., 1990. Constructibility and Mathematical Existence, Clarendon Press, Oxford.

[8] Cohen, P., 1963-1964. "The independence of the Continuum Hypothesis", I, PNAS, 50 (1963) 1143-1148 and II, PNAS, 51 (1964) $105-110$.

[9] Cohen, P. 1966. Set Theory and the Continuum Hypothesis, Benjamin, New York.

[10] Dehornoy, P., 1989. "La détermination projective d'après Martin, Steel et Woodin", Séminaire Bourbaki, \#710. 
[11] Dehornoy, P., 2003. "Progrès récents sur l'hypothèse du continu (d'après Woodin)", Séminaire Bourbaki, \#915.

[12] Dehornoy, P., 2007. "Au-delà du forcing: la notion de vérité essentielle en théorie des ensembles", Logique, dynamique et cognition, (J.B. Joinet, ed.), Publications de la Sorbonne, Paris, 147-169.

[13] Easton, W. B., 1970. "Powers of regular cardinals", $A M L, 1$ (1970), 139-178 (abridged version of the $1964 \mathrm{Ph} . \mathrm{D}$. thesis).

[14] Farah, I., Larson, P. B., 2005. "Absoluteness for Universally Baire Sets and the Uncountable, I", Quaderni di Matematica, 17 (2006), 47-92.

[15] Feferman S., 1989. "Infinity in Mathematics: Is Cantor Necessary?", Philosophical Topics, XVII, 2, 23-45.

[16] Feng, Q., Magidor, M., Woodin, W. H., 1992. "Universally Baire Sets of Reals", STC 1992, 203-242.

[17] Foreman, M., Magidor, M., Shelah, S., 1986. "0\# and Some Forcing Principles", Journal of Symbolic Logic, 51 (1986), 39-47.

[18] Foreman, M., 1998. "Generic large cardinals: new axioms for mathematics?", ICM, Vol. II, Berlin.

[19] Foreman, M., 2003. "Has the Continumm Hypothesis been http://www.math.helsinki.fi/logic/LC2003/presentations.

[20] Gödel, K., 1938. "The consistency of the axiom of choice and the generalized continuum hypothesis", PNAS, 25 (1938), 556-557.

[21] Gödel, K., 1940. "The consistency of the axiom of choice and of the generalized continuum hypothesis with the axioms of set theory", Annals of Math. Studies, Princeton University Press, Princeton.

[22] Gödel K., 1947. "What is Cantor's Continuum Problem", American Mathematical Monthly, 54 (1947), 515-545 (reprinted in Benacerraf-Putnam [1964], 470-485).

[23] Grigorieff, S., 1976. "Détermination des jeux boréliens d'après Martin", Séminaire Bourbaki, \#478.

[24] Husserl, E., 1900-1901. Logische Untersuchungen, Max Niemeyer, Halle (1913).

[25] Jackson, S., 1989. " $A D$ and the very fine structure of $L(\mathbb{R})$ ", Bulletin of the American Mathematical Society, 21, 1 (1989) 7781. 
[26] Jech, T., 1978. Set Theory, Academic Press, San Diego.

[27] Kanamori, A., 1995. The Higher Infinite, Perspectives in Mathematical Logic, Springer-Verlag, Berlin-Heidelberg-New York.

[28] Kant, I. Kants gesammelte Schriften, Preussische Akademie der Wissenschaften, Georg Reimer, Berlin, 1911.

[29] Maddy, P., 1988. "Believing the Axioms I, II", The Journal of Symbolic Logic, 53, 2 (1988), 481-511 ; 53, 3 (1988), 736-764.

[30] Maddy, P., 2005. "Mathematical existence", The Bulletin of Symbolic Logic, 11, 3 (2005), 351-376.

[31] Martin, Donald, 1975. "Borel Determinacy", Annals of Mathematics, 102 (1975), 363-371.

[32] Martin, David, 2001. Characterizations of $0^{\#}$, Master Thesis, Carnegie Mellon University.

[33] Martin, D., Steel, J., 1989. "A Proof of Projective Determinacy", Journal of the American Mathematical Society, 2, 1 (1989), 71125.

[34] Moschovakis, Y., 1980. Descriptive Set Theory, North-Holland.

[35] Panza, M., 1992. "De la Continuité comme Concept au Continu comme Objet", Le Labyrinthe du Continu, (J.-M. Salanskis, H. Sinaceur, eds.), Springer, Paris, 16-30.

[36] Peirce, C. S., 1960. Collected Papers, (C. Hartshorne and P. Weiss, eds.), The Belknap Press of Harvard University Press, Cambridge, Mass.

[37] Peirce, C. S., 1976. New elements of mathematics, (C. Eisele, ed.), Mouton Publishers, The Hague.

[38] Petitot, J., 1979. "Infinitesimale", Enciclopedia Einaudi, VII, 443-521, Einaudi, Torino.

[39] Petitot J., 1989. "Rappels sur l'Analyse non standard", La mathématique non-standard, (H. Barreau, J. Harthong, eds.), Editions du CNRS, Paris, 187-209.

[40] Petitot J., 1991. "Idéalités mathématiques et Réalité objective. Approche transcendantale", Hommage à Jean-Toussaint Desanti, (G. Granel ed.), 213-282, Editions TER, Mauvezin.

[41] Petitot J., 1992. "Continu et Objectivité. La bimodalité objective du continu et le platonisme transcendantal", Le Labyrinthe du 
Continu, (J.-M. Salanskis, H. Sinaceur, eds.), Springer-Verlag, Paris, 239-263.

[42] Petitot J., 1994. "Phenomenology of Perception, Qualitative Physics and Sheaf Mereology", Philosophy and the Cognitive Sciences, (16th Wittgenstein Symposium, R. Casati, B. Smith, G. White, eds), Verlag Hölder-Pichler-Tempsky, Vienna, 387-408.

[43] Petitot J., 1995. "Pour un platonisme transcendantal", L'objectivité mathématique. Platonisme et structures formelles, (M. Panza, J-M. Salanskis, eds), 147-178, Masson, Paris.

[44] Schimmerling, E., 2001. "The ABC's of Mice", The Bulletin of Symbolic Logic, 7, 4 (2001), 485-503.

[45] Solovay, R. M., 1971. "Real-Valued Measurable Cardinals", $A x$ iomatic Set Theory, (D. Scott ed.), Proceedings of Symposia in Pure Mathematics, Vol. XIII, Providence, AMS, 397-428.

[46] STC, 1992. Set Theory of the Continuum, (H. Judah, W. Just, H. Woodin, eds.), Springer, Berlin-New York.

[47] Steel, J., 2000. "Mathematics Needs New Axioms", http://math.berkeley.edu/ steel.

[48] Steel, J., 2001. "Inner Model Theory", to appear in the Handbook of Set Theory.

[49] Steel, J., 2004. "Generic absoluteness and the continuum prob-

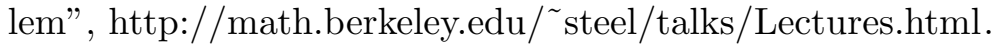

[50] Stern, J., 1976. "Le problème des cardinaux singuliers d'après Jensen et Silver", Séminaire Bourbaki, \#494.

[51] Stern, J., 1984. "Le problème de la mesure", Séminaire Bourbaki, \#632.

[52] Thom, R., 1992. "L'Antériorité ontologique du Continu sur le Discret", Le Labyrinthe du Continu, (J.-M. Salanskis, H. Sinaceur, eds.), Springer, Paris, 137-143.

[53] Weyl, H., 1918. Das Kontinuum. Kritishe Untersuchungen über die Grundlager der Analysis, Veit, Leipzig, (English translation, Lanham, University Press of America, 1987).

[54] Woodin, W. H., 1999. The Axiom of Determinacy, Forcing Axioms, and the Nonstationary Ideal, De Gruyter, Berlin.

[55] Woodin, W. H., 2000. "The Continuum Hypothesis", Logic Colloquium, Paris. 
[56] Woodin, W. H., 2001a. The continuum hypothesis, I-II, Notices Amer. Math. Soc., 48, 6 (2001), 567-576 and 48, 7 (2001) 681-690.

[57] Woodin, W. H., 2001b. "The $\Omega$ Conjecture", Aspects of Complexity, De Gruyter, Berlin, 155-169.

[58] Woodin, W. H., 2002. "Beyond $\Sigma_{1}^{2}$ Absoluteness", ICM 2002, Vol. I, 515-524.

[59] Woodin, W. H., 2003. "Set theory after Russell: the journey back to Eden", One Hundred Years of Russell's Paradox, (G. Link, ed.), De Gruyter, Berlin, 29-47 (2004).

[60] Woodin, W. H., 2004. "Is there really any evidence that the Continuum Hypothesis has no answer?", http://www.lps.uci.edu/home/conferences/ LagunaWorkshops/LagunaBeach2004/irv.pdf 